\title{
Large Scale DD Simulation Results for Crystal Plasticity Parameters in Fe-Cr and Fe-Ni Systems
}

\section{Nuclear Energy Enabling Technology}
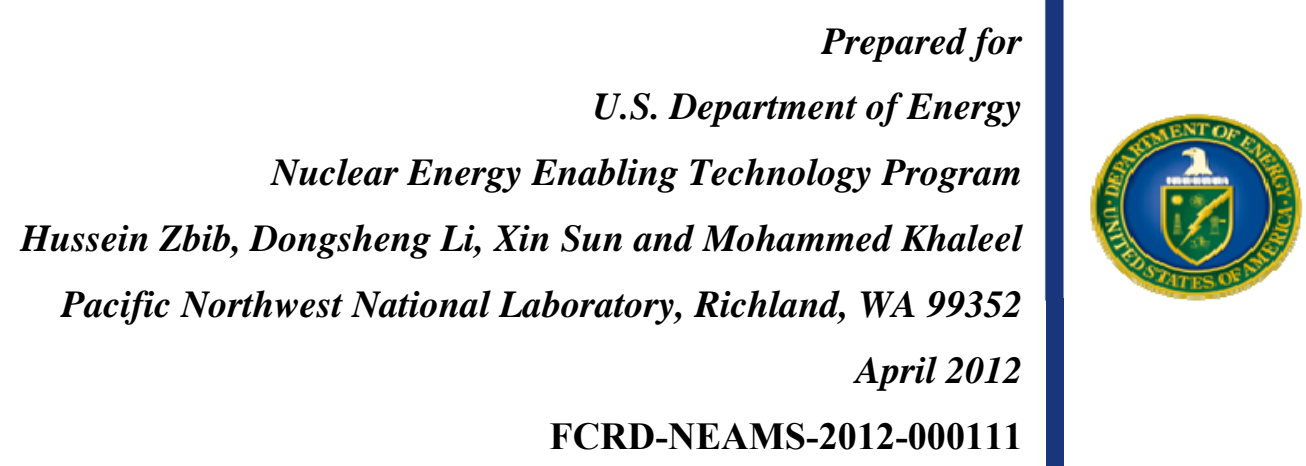

PNNL-21334 


\section{DISCLAIMER}

This information was prepared as an account of work sponsored by an agency of the U.S. Government. Neither the U.S. Government nor any agency thereof, nor any of their employees, makes any warranty, expressed or implied, or assumes any legal liability or responsibility for the accuracy, completeness, or usefulness, of any information, apparatus, product, or process disclosed, or represents that its use would not infringe privately owned rights. References herein to any specific commercial product, process, or service by trade name, trade mark, manufacturer, or otherwise, does not necessarily constitute or imply its endorsement, recommendation, or favoring by the U.S. Government or any agency thereof. The views and opinions of authors expressed herein do not necessarily state or reflect those of the U.S. Government or any agency thereof. 


\section{Reviewed by:}

National Technical Director, Nuclear energy

Advanced Modeling and Simulation

Keith Bradley Date

\section{Concurred by:}

Acting Director, Advanced Modeling and

Simulation Office

Trevor Cook Date

\section{Approved by:}

Deputy Assistant Secretary, Nuclear energy

John Kelly Date




\section{SUMMARY}

The development of viable nuclear energy source depends on ensuring structural materials integrity. Structural materials in nuclear reactors will operate in harsh radiation conditions coupled with high level hydrogen and helium production, as well as formation of high density of point defects and defect clusters, and thus will experience severe degradation of mechanical properties. Therefore, the main objective of this work is to develop a capability that predicts aging behavior and in-service lifetime of nuclear reactor components and, thus provide an instrumental tool for tailoring materials design and development for application in future nuclear reactor technologies. Towards this end goal, the long term effort is to develop a physics-based multiscale modeling hierarchy, validated and verified, to address outstanding questions regarding the effects of irradiation on materials microstructure and mechanical properties during extended service in the fission and fusion environments. The focus of the current investigation is on modern steels for use in nuclear reactors including high strength ferritic-martensitic steels (Fe-Cr-Ni alloys).

The effort is to develop a predictive capability for the influence of irradiation on mechanical behavior. Irradiation hardening is related to structural information crossing different length scales, such as composition, dislocation, and crystal orientation distribution. To predict effective hardening, the influence factors along different length scales should be considered. Therefore, a hierarchical upscaling methodology is implemented in this work in which relevant information is passed between models at three scales, namely, from molecular dynamics to dislocation dynamics to dislocation-based crystal plasticity. The molecular dynamics (MD) was used to predict the dislocation mobility in body centered cubic (bcc) Fe and its $\mathrm{Ni}$ and $\mathrm{Cr}$ alloys. The results are then passed on to dislocation dynamics to predict the critical resolved shear stress (CRSS) from the evolution of local dislocation and defects.

In this report the focus is on the results obtained from large scale dislocation dynamics simulations. The effects of defect density and materials structure were investigated while new evolution laws were proposed. These results will form the bases for the development of evolution and hardening laws for a dislocation-based crystal plasticity framework. The hierarchical upscaling method being developed in this project can provide a guidance tool to evaluate performance of structural materials for nextgeneration nuclear reactors. Combined with other tools developed in the Nuclear Energy Advanced Modeling and Simulation (NEAMS) program, the models developed will have more impact in improving the reliability of current reactors and affordability of new reactors. 


\section{CONTENTS}

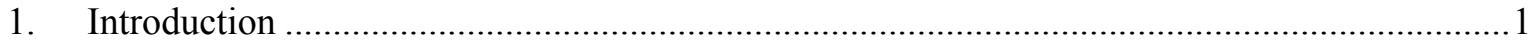

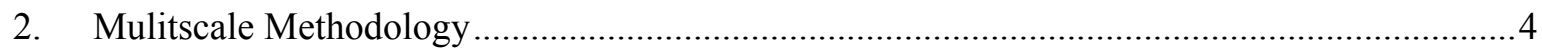

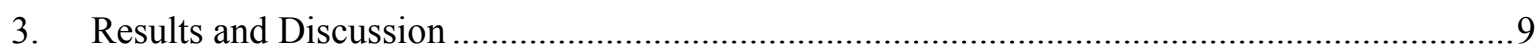

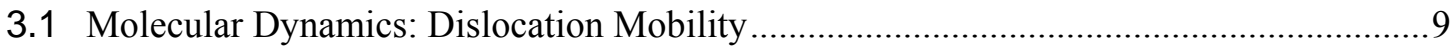

3.2 Discrete Dislocation Dynamics: Irradiation Induced Hardening …............................... 10

3.3 Continuum Dislocation Dynamics - Crystal Plasticity ...............................................22

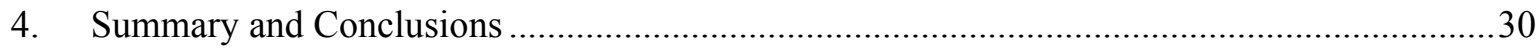

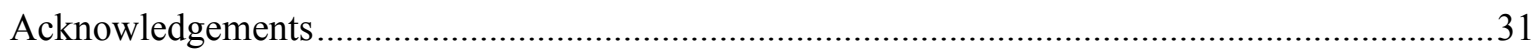

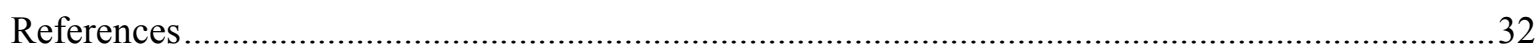




\section{LIST OF FIGURES}

Figure 1. Dislocation mobility as a function of the temperature in (a) Fe-Ni and (b) Fe-Cr systems. In both cases the concentration of nickel or chromium was varied from $5 \%$ to $20 \% . .7$

Figure 2 Typical dislocation dynamics simulation: a) Simulation cell with initial random distribution of Frank-Read dislocation sources and FS loops, b) dislocation-defect structure after plastic deformation, c) a close-up view showing the interaction between dislocations and FS loops, d) underlying dislocation structure resulting from cross-slip, e) FS loops structure showing the formation of defect-free channels

Figure 3. DD simulation results of Fe-Ni alloys: Stress-strain curves for various Ni concentrations and irradiation defect density.....

Figure 4. DD simulation results of Fe-Cr alloys: Stress-strain curves for various $\mathrm{Cr}$ concentrations and irradiation defect density.

Figure 5. Fe-\%Ni DD results: Evolution of defect density for various Ni concentrations and irradiation defect density

Figure 6. Fe-\%Ni DD results: Evolution of dislocation density for various Ni concentrations and irradiation defect density.

Figure 7. Fe-\%Ni- Dislocation dynamics results: Effect of Ni concentrations ..............................15

Figure 8. The dislocation mobility and CRSS for Fe-NI and $\mathrm{Fe}-\mathrm{Cr}$ systems with no defects. .........16

Figure 9. Increase of the CRSS with irradiation defect density in $\mathrm{Fe}-\mathrm{Ni}$....................................... 18

Figure 10. Increase of the CRSS with irradiation defect density in Fe-Cr....................................19

Figure 11. Evolution of defect density as a function plastic strain for $\mathrm{Fe}-10 \% \mathrm{Ni}$.......................22

Figure 12. Measured and predicted stress strain curve of single crystal $\alpha-\mathrm{Fe}$ under uniaxial tension along $<001>$ direction.

Figure 13. Measured and predicted stress strain curve of single crystal $\alpha$-Fe under uniaxial tension along $<011>$ direction. 


\section{LIST OF TABLES}

Table 1. Simulated mobility (in $1 / \mathrm{Pa} \cdot \mathrm{s}$ ) of an edge dislocation inside various Fe alloys at various temperatures.

Table 2. Elastic properties for Fe-Cr and Fe-Ni alloys calculated using the rule of mixture............ 9

Table 3. The coefficients of the curve fitting using a power function for various concentrations ... 10

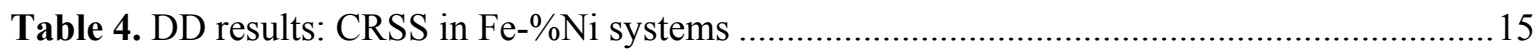

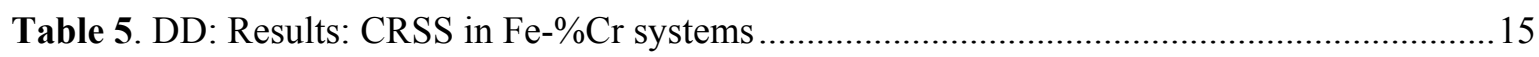

Table 6. List of parameters used in the continuum dislocation dynamics model............................28 


\section{ACRONYMS}

bcc body-centered cubic

$\mathrm{Cr}$

chromium

CRSS critical resolved shear stress

MD molecular dynamics

DD dislocation dynamics

$\mathrm{CP} \quad$ crystal plasticity

DOE U.S. Department of Energy

fcc face-centered cubic

$\mathrm{Fe} \quad$ iron

FS Frank sessile

$\mathrm{Ni} \quad$ nickel

PNNL Pacific Northwest National Laboratory

R\&D research and development 


\section{FUEL CYCLE R\&D PROGRAM \\ Large scale DD simulation results for crystal plasticity parameters in $\mathrm{Fe}-\mathrm{Cr}$ and Fe-Ni systems}

\section{INTRODUCTION}

The development of new-generation nuclear reactors depends on the availability of materials that can operate safely in severe environments for an extended service lifetime [1-2]. Materials operating in such a harsh environment are subjected to high doses of irradiation which causes changes in microstructure. These changes are responsible for dimensional instabilities, such as swelling and irradiation creep, and mechanical property evolution and degradation, such as irradiation hardening and post-yield deformation behavior including plastic flow and subsequent localization, which impact component performance and reliability.

The evolution of microstructural features with irradiation dose and temperature involves coalescence of vacancies and interstitials into voids and dislocation loops that cause swelling. In steels, void swelling can occur at temperatures up to about $800 \mathrm{~K}$ [3-4]. Modern steels used in nuclear reactors include high strength, ferritic, martensitic and oxide dispersion strengthened steels. These steels reduce the occurrence of void swelling and are more stable in the presence of defects that arise due to their severe functional environment. These steels are used in conditions subjected to creep and stress corrosion in the reactor environment that adversely affects their useful service life. Although ferritic-martensitic steels are quite resistant to swelling and maintain good fracture toughness at irradiation above $673 \mathrm{~K}[5-6]$, they are prone to loss of ductility at lower irradiation temperatures [7-8].

Over the past two decades, significant advances have been made in understanding the effects of irradiation on materials microstructure ${ }^{\mathrm{a}}$ and mechanical properties by focusing theory,

\footnotetext{
a This includes crystal lattice defects, such as dislocations, voids and grain boundaries, as well as microchemical arrangements, including second phase precipitates and regions of solute segregation that extend from the $\mathrm{nm}$ to $\mathrm{mm}$ scale.
} 
experiments and modeling on the basic underlying physical mechanisms [9-23]. For example, it is well established that the effect of irradiation on ferritic-martensitic alloys at low to intermediate temperatures is increased yield stress, reduced strain hardening capacity, and flow localization at lower strains [24-25]. Furthermore, the predominant microstructural features include dislocation loops, voids, regions of solute segregation and second-phase precipitates. The initial density and evolution of these features depend on some key variables, such as irradiation temperature, dose and dose rate, helium production rate, and alloy composition. The mechanical properties depend on the interaction of dislocations with the defects as well as the interaction between dislocations. However, predicting the balance of these features based on key variables and detailed mechanisms of their formation, as well as their precise character and composition, remains an outstanding question. A firm understanding of the fundamentals of defect production and evolution (at the nano and micro scales) in an alloy and its effect on macroscopic mechanical properties is essential for evaluating the material stability of candidate engineering materials in experimentally uncharted irradiation conditions. Moreover, current models that address defect production are mostly limited to pure metals, for example $\mathrm{Fe}, \mathrm{Pd}$, and $\mathrm{Cu}$. However, production and evolution of point defects and defect clusters and their interaction with grain boundaries and dislocations are quite different in alloys compared to pure metals [26-27]. This may be attributed to the manner in which point defects interact within the displacement cascade and with solute atoms, which in turn affect point-defect mobility, cluster formations, and dislocation-defect-grain boundary interaction. These all influence strength, ductility and failure. This work addresses outstanding issues related to the effects of irradiation in ferritic-martensitic steels by focusing on two key scientific issues: 1) the effect of alloy chemistry and solute-defect interactions on microstructural evolution with emphasis on $\mathrm{Fe}-\mathrm{Cr}, \mathrm{Fe}-\mathrm{Ni}$, and $\mathrm{Fe}-\mathrm{Cr}-\mathrm{Ni}$ systems and 2) linking the underlying materials microstructure to mechanical properties, including yield stress and postyield deformation behavior.

The effect of irradiation on materials microstructure and properties is a classic example of an inherently multiscale phenomenon, involving processes spanning a range of length and time scales. At the smallest scales (nanometer and picoseconds), high-energy particle collisions with lattice atoms result in displacement cascades that produce highly non-equilibrium point defects (vacancies and interstitials) and point defect cluster concentrations with a high degree of spatial correlation. These defects and clusters diffuse over macroscopic length and time scales, 
significantly altering the material's chemistry and microstructure. Computer simulation and modeling can provide significant insight toward understanding the effects of irradiation on materials and potentially lead to the development of new-generation materials. Due to the costs of developing and testing such new materials, using computational techniques has been proven to be highly preferable and cost effective. The application of first principle, molecular (MD), and dislocation dynamics (DD) is a paradigm of techniques employed to model the behavior of prospective materials in future nuclear energy systems [28-30]. Previous empirical models are being replaced by more physical ones that take advantage of new results at the microscopic level, as well as the tremendous improvements in computing science, and breakthroughs in physical metallurgy at the atomic scale. However, predicting material behavior based on this knowledge still is an unsolved problem, mainly due to its multiscale nature involving processes spanning diverse length and time scales. Current models that address the issue of defect production and interaction are limited to one scale only without any interaction between the different scales. At the smallest scales (nanometer and picoseconds), irradiation dose and temperature cause the coalescence of vacancies and interstitials into voids and dislocation loops. These defects and clusters diffuse over macroscopic length and time scales, altering significantly the chemistry and microstructure of the material. In order to effectively predict the mechanical behavior of the materials under irradiation at the continuum scale, critical information should be determined and progressively passed from one scale to another.

In the heart of any macroscopic model is the collective behavior of dislocations inside the material under study. Among the available computational techniques, discrete dislocation dynamics is the most commonly used to study the materials behavior at the mesoscale.

Discrete dislocation dynamics (DD) is a powerful tool that has been advanced significantly in the past decade [31-34]. It has been used to explain the effect of irradiation on mechanical properties through large scale simulations of interaction of numerous numbers of dislocations with defect clusters. The work of Zbib and co-worker have shown that dislocations interaction with the elastic fields of nanosize defect clusters in $\mathrm{Cu}$ and $\mathrm{Pd}$ lead to hardening followed by localized deformation and channel formation resulting from defect cluster annihilation by dislocations [30, 35-41]. In the present work we investigate the effect of irradiation hardening in $\mathrm{Fe}-\mathrm{Cu}-\mathrm{Ni}$ systems. The goal is to predict the stress-strain curve and the 
critical resolved shear stress as a function of defect density, which is then used in the crystal plasticity model. However, in order for this method to work, a detailed knowledge of the dislocations mobility in an analytical form inside the materials is required. In the literature various studies can be found to deal with the dislocation mobility using molecular dynamics simulations. However, they are all limited to simple systems and they do not attempt to calculate the effect of the concentration on the mobility [42-44]. Recently work has been done in calculating the mobility of screw dislocations in $\alpha$-iron and to derive analytical expressions for the use within the dislocation dynamics [45]. Although the motion of screw dislocations is dominant in the deformation of bcc structures, the role of edge dislocations is not insignificant. Furthermore, in the dislocation dynamics framework, the mobility of the screw dislocations can be calculated as a fraction of the mobility of the edge counterpart $[37,46]$. In view of this, molecular dynamics simulations were performed in iron alloys with various concentrations of nickel and chromium [47-48]. This system was chosen because of the availability of the interatomic potentials and the similarity of the systems considered for the next generation nuclear reactors. Due to the generality of the technique, other material systems can be studied as well. This information, in conjunction with the information described elsewhere [49] can be used to study the degradation of the material at the microscale. In this report, first the calculated dislocation mobility is used to develop rules of the mobility as function of temperature and concentration in a suitable form for the dislocation dynamics framework. Then the results gathered from the DD simulations are presented followed by their analysis that leads to the evaluation of the critical resolved shear stress in $\mathrm{Fe}-\mathrm{Ni}-\mathrm{Cu}$ alloys as a function of concentration, as well as irradiation damage. Finally, semi-empirical expressions are developed for future use in a dislocation-based crystal plasticity framework, followed by discussion and the conclusions of this work.

\section{MULTISCALE METHODOLOGY}

The modeling methodology involves a hierarchical approach integrating molecular dynamics (MD) simulations, dislocation dynamics (DD) simulations, and crystal plasticity with internal variable theory, over the relevant length and time scales to model the fates of defects and solutes and thus, predict microstructural evolution in irradiated ferritic/martensitic steels. The evolution 
of the microstructure under irradiation will be linked to a quantitative prediction of irradiation hardening and post-yield deformation behavior through the use of dislocation dynamics (DD) simulations, crystal plasticity and continuum internal variable models. The following procedure is a summary of the multiscale approach that we have adopted.

a) MD simulations: Dislocation mobility and defect properties in $\mathrm{Fe}$-alloys ( $\mathrm{Fe}-\mathrm{Cr}$, and $\mathrm{Fe}-\mathrm{Ni}$ ) are obtained from atomistic simulations based on semi-empirical EAM and/or MEAM potentials. Specifically, defect cluster energetics, physical insight into kinetic processes and interactions are obtained from large-scale atomistic simulations, including the fate of moving defect-solute clusters with dislocations and grain boundaries, the mechanisms of moving dislocation interactions with dislocation loops, defect clusters and second phase particles.

b) DD-MD Linkage to Mechanical Property Changes: The dislocation mobility and microstructure predicted by the atomistic simulations serves as input to three-dimensional DD simulation to quantitatively predict radiation-induced mechanical property changes. Specifically, MD would lead information about defect density evolution in the bulk and at grain boundaries, defect-dislocation-solute interaction and conditions for annihilation of defects, pinning and dislocation climb, as well as the dislocation mobility and its dependence on solute concentration.

c) MD-Informed Dislocation Dynamics Simulations: The collective behavior of an ensemble of dislocations in a crystal is modeled by calculating the forces on each dislocation and taking into account all possible reactions among dislocations, as well as between network of dislocations, and the radiation-induced defect microstructure. A key component to quantitative 3D-DD simulations is to understand the mechanisms and features of a variety of nanostructural features which interact with moving dislocations, are obtained from large-scale MD simulations.

d) DD-Crystal Plasticity Linkage to Mechanical Property Changes: DD provides quantitative data for the dependence of flow stress on defect cluster density and evolution of cluster density as a function of plastic strain which can be used to develop models for use in crystal plasticity. 
e) DD-Informed Crystal Plasticity (CP): Crystal plasticity framework will include information passed from DD and MD. Hardening laws will be modified to include the effect of defect cluster as determined by DD, and the properties of the grain boundaries as determined by MD.

The focus of the current report is on results from large scale dislocation dynamics simulations with input from molecular dynamics. The results from molecular dynamics simulations for dislocation mobility and dislocation interaction with voids and precipices in iron alloys with various concentrations of nickel and chromium are reported in [47-48, 50-51]. The Embedded Atom Method (EAM) [52] and potentials developed in [53-55] for the pure iron, Fe$\mathrm{Cr}$ and Fe-Ni respectively were used. The results for the edge dislocation mobility are summarized in Table 1, and plotted in Figures 1a-b. These results are used in conjunction with the dislocation dynamics simulations discussed below, and will be further analyzed in the next section.

Table 1. Simulated mobility (in $1 / \mathrm{Pa} \cdot \mathrm{s}$ ) of an edge dislocation inside various $\mathrm{Fe}$ alloys at various temperatures.

\begin{tabular}{|c|c|c|c|c|c|c|c|}
\hline Temp & & & & & & & \\
\hline Struct. & $10 \mathrm{~K}$ & $50 \mathrm{~K}$ & $100 \mathrm{~K}$ & $150 \mathrm{~K}$ & $200 \mathrm{~K}$ & $250 \mathrm{~K}$ & $300 \mathrm{~K}$ \\
\hline$\alpha-\mathrm{Fe}$ & $3.55 E+03$ & $2.93 E+03$ & $2.61 E+03$ & $2.50 \mathrm{E}+03$ & $2.43 E+03$ & $2.38 E+03$ & $2.38 \mathrm{E}+03$ \\
\hline $\mathrm{Fe}-5 \% \mathrm{Ni}$ & $4.31 E+03$ & $4.08 E+03$ & $4.02 E+03$ & $3.68 \mathrm{E}+03$ & $3.73 E+03$ & $3.79 \mathrm{E}+03$ & $2.79 E+03$ \\
\hline $\mathrm{Fe}-10 \% \mathrm{Ni}$ & $5.05 E+03$ & $4.37 E+03$ & $4.13 E+03$ & $4.07 E+03$ & $4.07 E+03$ & $4.31 E+03$ & $3.94 E+03$ \\
\hline $\mathrm{Fe}-15 \% \mathrm{Ni}$ & $5.43 E+03$ & $4.41 E+03$ & $4.39 E+03$ & $4.85 E+03$ & $5.21 E+03$ & $5.56 \mathrm{E}+03$ & $5.00 \mathrm{E}+03$ \\
\hline $\mathrm{Fe}-20 \% \mathrm{Ni}$ & $5.99 \mathrm{E}+03$ & $4.52 E+03$ & $4.65 E+03$ & $5.03 E+03$ & $5.29 \mathrm{E}+03$ & $5.29 \mathrm{E}+03$ & $5.18 \mathrm{E}+03$ \\
\hline $\mathrm{Fe}-5 \% \mathrm{Cr}$ & $3.48 E+03$ & $2.82 E+03$ & $2.67 E+03$ & $2.55 E+03$ & $2.56 \mathrm{E}+03$ & $2.60 E+03$ & $2.71 E+03$ \\
\hline $\mathrm{Fe}-10 \% \mathrm{Cr}$ & $3.57 E+03$ & $3.08 \mathrm{E}+03$ & $2.95 E+03$ & $3.07 E+03$ & $3.12 E+03$ & $3.18 E+03$ & $3.15 E+03$ \\
\hline $\mathrm{Fe}-15 \% \mathrm{Cr}$ & $3.68 \mathrm{E}+03$ & $3.09 E+03$ & $2.92 E+03$ & $2.72 E+03$ & $2.84 E+03$ & $2.78 E+03$ & $3.13 E+03$ \\
\hline $\mathrm{Fe}-20 \% \mathrm{Cr}$ & $2.98 \mathrm{E}+03$ & $2.73 \mathrm{E}+03$ & $2.59 E+03$ & $2.69 \mathrm{E}+03$ & $2.82 \mathrm{E}+03$ & $2.83 \mathrm{E}+03$ & $3.09 \mathrm{E}+03$ \\
\hline
\end{tabular}

A complete description of the DD method and its extension to dislocations in heterogeneous media can be found in [56]. According to the theory the motion of a dislocation segment is governed by a Newtonian-type equation of motion with a driving force that consists of a number of components, including Peierls lattice friction forces, dislocation-dislocation interaction forces through their stress fields, forces due to externally applied forces, osmotic climb forces, images forces due to free surfaces or from differences in elastic moduli for dislocations in heterogamous media and near interfaces, etc. In the case of irradiation damage, dislocations also interact with defect clusters whose density in bcc metals can be on the order of 
$10^{24} \mathrm{~m}^{-3}$ for a variety of neutron irradiated metals near room temperature. In bcc metals defects are Frank-Sessile (FS) dislocation loops of $\mathrm{a}<100>$ and $\mathrm{a} / 2<111>$, in almost equal proportion [57], and the radius varies between $1 \mathrm{~nm}$ to $3 \mathrm{~nm}$. In DD, the elastic interaction and short-range interaction between dislocations and FS loops is computed explicitly based on the work of Khraishi et. al. [58], which was originally developed for face centered cubic (fcc) systems. Each defect possesses its own stress field which contributes to the Peach-Koehler force, impeding the motion of the dislocation and contributing to irradiation hardening.

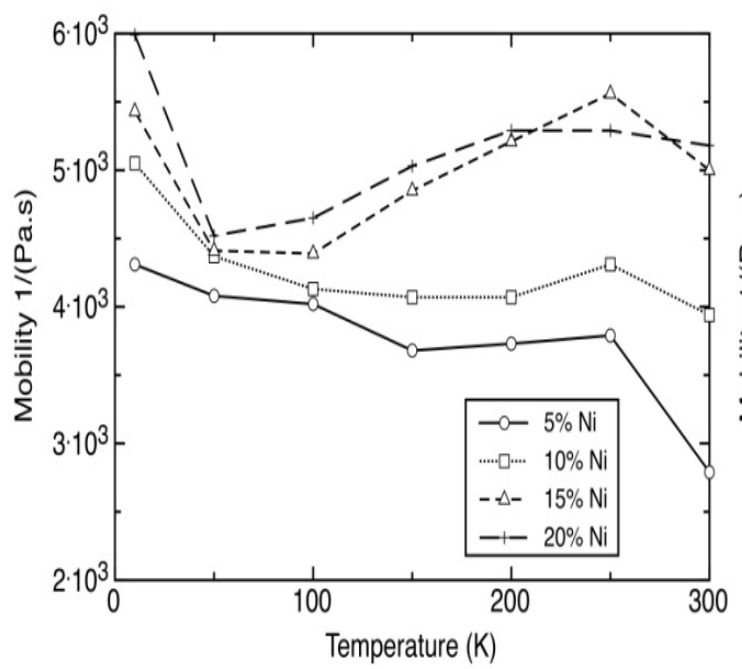

(a)

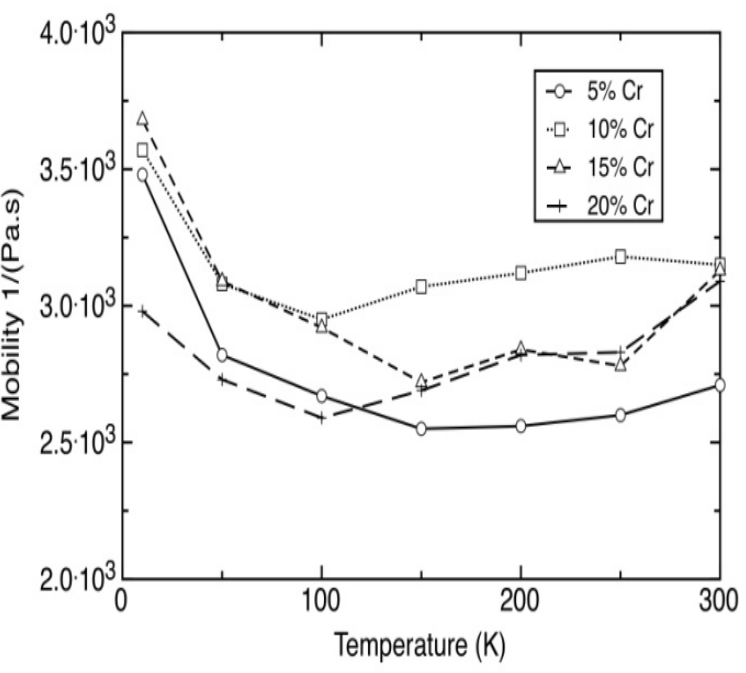

(b)

Figure 1. Dislocation mobility as a function of the temperature in (a) $\mathrm{Fe}-\mathrm{Ni}$ and (b) $\mathrm{Fe}-\mathrm{Cr}$ systems. In both cases the concentration of nickel or chromium was varied from $5 \%$ to $20 \%$.

Herein, we investigated the effect of irradiation hardening in bcc with the goal to predict the stress-strain curve and critical resolved shear stress as a function of alloy and defect density, to be used in the crystal plasticity model. The DD simulation unit cell, shown in Figure 2a, is a $4.5 \times 4.5 \times 5.97 \mu \mathrm{m}^{3}$ cube cell that contains an initial density of Frank-Read sources distributed randomly on the primary [59] slip planes. In the simulations, periodic boundary conditions were imposed. The cell is loaded in tension with a constant strain rate of 100/s. The effect of irradiation is accounted for by mapping into the DD box a spatial distribution of FS loops with density ranging from $10^{20} / \mathrm{m}^{3}$ to $10^{24} / \mathrm{m}^{3}$. The loops are 1 to 3 nanometers in radius, and the radius is randomly generated to fall within the specified interval. The model also generates 
Frank-Read sources, represented as finite dislocation segments pinned at ends, lying on $\{110\}$ glide planes, and Burgers vectors of the type $<111>$.

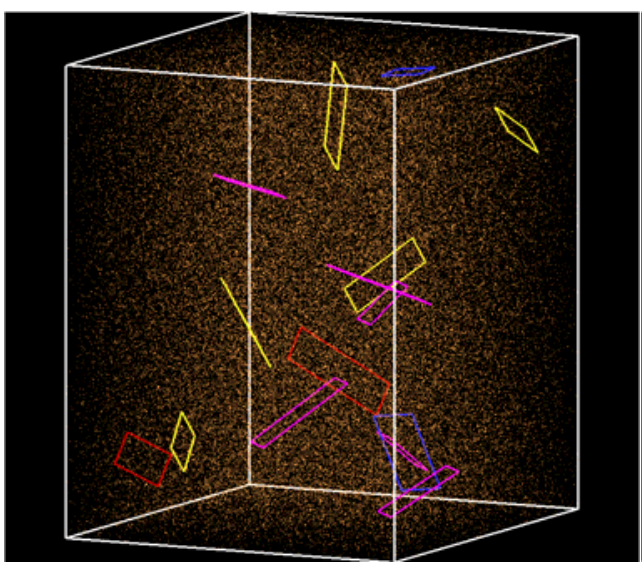

a)

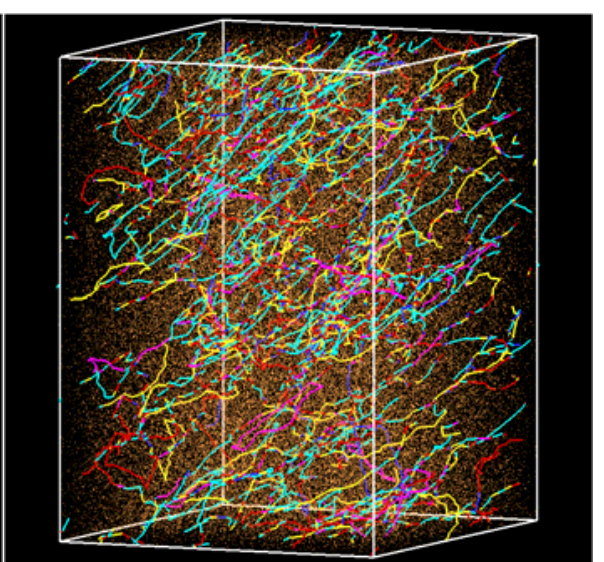

b)

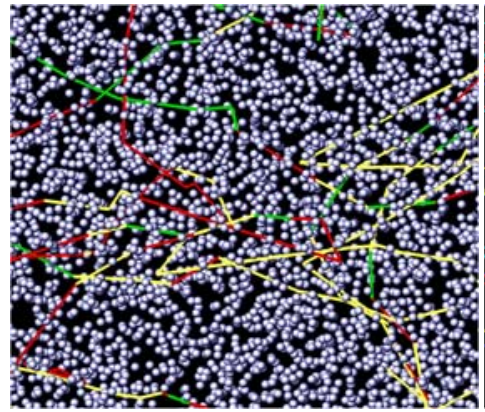

c)

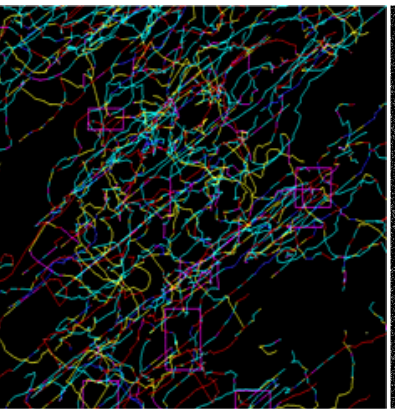

d)

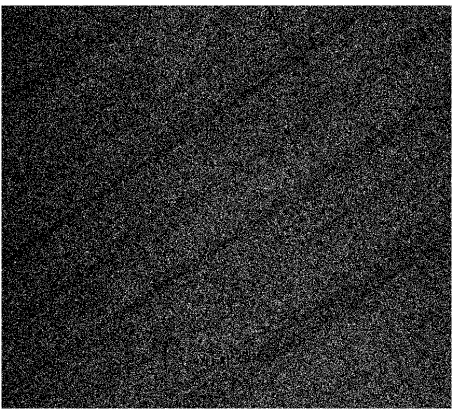

e)

Figure 2 Typical dislocation dynamics simulation: a) Simulation cell with initial random distribution of Frank-Read dislocation sources and FS loops, b) dislocation-defect structure after plastic deformation, c) a close-up view showing the interaction between dislocations and FS loops, d) underlying dislocation structure resulting from cross-slip, e) FS loops structure showing the formation of defect-free channels.

The DD simulations were performed for all the $\mathrm{Fe}-\mathrm{Cr}$ and $\mathrm{Fe}-\mathrm{Ni}$ cases at $\mathrm{T}=300 \mathrm{~K}$. In the DD simulations, the required material parameters are: the shear modulus $\mu$, the Poisson's ratio $v$, the dislocation mobility $M$, and the Burgers vector magnitude $b$. For the Fe-Ni and Fe-Cr alloys under consideration, the elastic properties are listed in Table 2, which are determined using a simple rule of mixture. The dislocation mobility for the edge or mixed dislocation we use the values given in Table 1 , and $b=0.2482 \mathrm{~nm}$. The screw dislocations mobility can be calculated as a fraction of the mobility of the edge counterpart as suggested in $[37,46]$. Here we assume 
that the screw component's mobility is proportional to that of the edge with a proportionality factor equal to $10^{-3}$.

Table 2. Elastic properties for $\mathrm{Fe}-\mathrm{Cr}$ and $\mathrm{Fe}-\mathrm{Ni}$ alloys calculated using the rule of mixture

\begin{tabular}{|c|c|c|}
\hline Elastic Properties & Shear Modulus (Gpa) & Poisson's ratio \\
\hline $\mathrm{a}-\mathrm{Fe}$ & 83 & 0.29 \\
\hline $\mathrm{Ni}$ & 76 & 0.31 \\
\hline $\mathrm{Cr}$ & 115 & 0.21 \\
\hline $\mathrm{Fe}-5 \% \mathrm{Ni}$ & 82.65 & 0.29 \\
\hline $\mathrm{Fe}-10 \% \mathrm{Ni}$ & 82.3 & 0.29 \\
\hline $\mathrm{Fe}-15 \% \mathrm{Ni}$ & 81.95 & 0.29 \\
\hline $\mathrm{Fe}-20 \% \mathrm{Ni}$ & 81.6 & 0.29 \\
\hline & & \\
\hline $\mathrm{Fe}-5 \% \mathrm{Cr}$ & 84.6 & 0.29 \\
\hline $\mathrm{Fe}-10 \% \mathrm{Cr}$ & 86.2 & 0.28 \\
\hline $\mathrm{Fe}-15 \% \mathrm{Cr}$ & 87.8 & 0.28 \\
\hline $\mathrm{Fe}-20 \% \mathrm{Cr}$ & 89.4 & 0.27 \\
\hline
\end{tabular}

\section{RESULTS AND DISCUSSION}

\subsection{Molecular Dynamics: Dislocation Mobility}

The atomistic simulations, reported in [47-48, 50-51], were performed for three iron systems: $\alpha-\mathrm{Fe}, \mathrm{Fe}-\mathrm{Ni}$ and $\mathrm{Fe}-\mathrm{Cr}$. For the Fe-Ni and Fe-Cr systems, four different concentrations of nickel and chromium $(5 \%, 10 \%, 15 \%$ and $20 \%)$ were considered to study the effect of the concentration on the dislocation mobility. The results are summarized in Table 1, and plotted in Figure 1. The results reveal that edge dislocation mobility is higher in the Fe-Ni systems compared to the $\alpha-\mathrm{Fe}$ and is increasing as the Ni concentration increases. On the other hand, the dislocation mobility inside the Fe-Cr is comparable to the pure iron although it still is about 5\% higher. The higher edge mobility of the alloys compared to the pure Fe is backed by experimental observations that show a higher density of screw dislocations in alloys because the edge dislocations are very fast and disappear at the surface of the specimen [60-61]. This also is backed by other simulations of screw dislocations mobility [10], showing that it is lower than the edge mobility. It seems that two lines with different slopes intersecting at $50 \mathrm{~K}$ can be used to approximate the dislocation mobility as a function of the temperature. These lines can be extended to predict the edge dislocation mobility at higher temperatures, eliminating the need to 
perform extended atomistic simulations over a broad range of temperatures (providing this range remains below the melting temperature). The predicted law for the mobility of an edge dislocation can complement similar results for the screw dislocations in Fe [10] to form a complete set of rules that can be used in DD to study the behavior of iron and iron alloys at the mesoscale. For more details on the implementation of this technique see [48-49]. Results for the edge dislocation mobility $M_{e}$ from the atomistic simulations can be fitted to a relation of the following form:

$$
M_{e}=a_{0}+m \sqrt{T-T_{0}}
$$

The values for the parameters appearing in equation (1) are shown in Table 3 for the Fe$\mathrm{Ni}$ and $\mathrm{Fe}-\mathrm{Cr}$ systems. In general, results show that the fitting parameters are either constant (the case of $T_{0}$ for both solutes and $m$ for the $\mathrm{Cr}$ ) or vary in a linear manner with the concentration that implies the existence of a general law for the mobility of edge dislocation as a function of the concentration in iron alloys that include $\mathrm{Ni}$ and $\mathrm{Cr}$. This law will follow the form described above with fitting parameters that will vary for each solute. This also leads to the conclusion that the edge dislocation mobility depends on the concentration of the $\mathrm{Ni}$ or $\mathrm{Cr}$ inside $\mathrm{Fe}$. Furthermore, the parameterization used in this work provides a good starting point to study the effect of the concentration on the Fe-Ni and Fe-Cr alloys in a form suitable for the DD framework.

Table 3. The coefficients of the curve fitting using a power function for various concentrations

\begin{tabular}{|c|c|c|c|c|c|c|c|}
\hline$\% \mathrm{Ni}$ & $\mathrm{a}_{0}$ & $\mathrm{~m}$ & $\mathrm{~T}_{0}$ & $\% \mathrm{Cr}$ & $\mathrm{a}_{0}$ & $\mathrm{~m}$ & $\mathrm{~T}_{0}$ \\
\hline 5 & 3771.43 & 437.49 & 1494.96 & 5 & 2769.99 & 321.65 & 1101.91 \\
\hline 10 & 4277.14 & 496.30 & 1694.38 & 10 & 3160.00 & 366.97 & 1254.08 \\
\hline 15 & 4978.57 & 577.15 & 1973.27 & 15 & 3022.85 & 350.86 & 1200.63 \\
\hline 20 & 5135.71 & 595.49 & 2036.13 & 20 & 2818.57 & 327.30 & 1122.48 \\
\hline
\end{tabular}

\subsection{Discrete Dislocation Dynamics: Irradiation Induced Hardening}

Results from a series of DD simulations, relevant to crystal plasticity, are summarized in Figures 2-7. The simulations were performed for a series of initial dislocation and defect densities. The defect densities are labeled for the corresponding values listed in Tables 4 and 5 . 
Figures 2a-e show a typical dislocation-defect structure after a certain amount of strain in the presence of FS loops. The dislocation structure shown in Figure $2 \mathrm{~b}$ and highlighted in Figure $2 \mathrm{~d}$ is composed mainly of extended dislocations of screw character, resulting from the fact that the mobility of the screw dislocation is three orders of magnitude less than the edge. Figure 2c shows the pinning effect of the FS loops resulting from the elastic interaction between the dislocations and the loops. As the dislocations sweep through the cloud of the FS loops, some of the loops get annihilated, depending on their interaction energy with the sweeping dislocations as discussed in $[36,41,59]$. This results in the formation of defect free channels as shown in Figure 2 and subsequently causes a drop in the flow stress as deduced from the stress-strain curves shown in Figures 3 and 4.
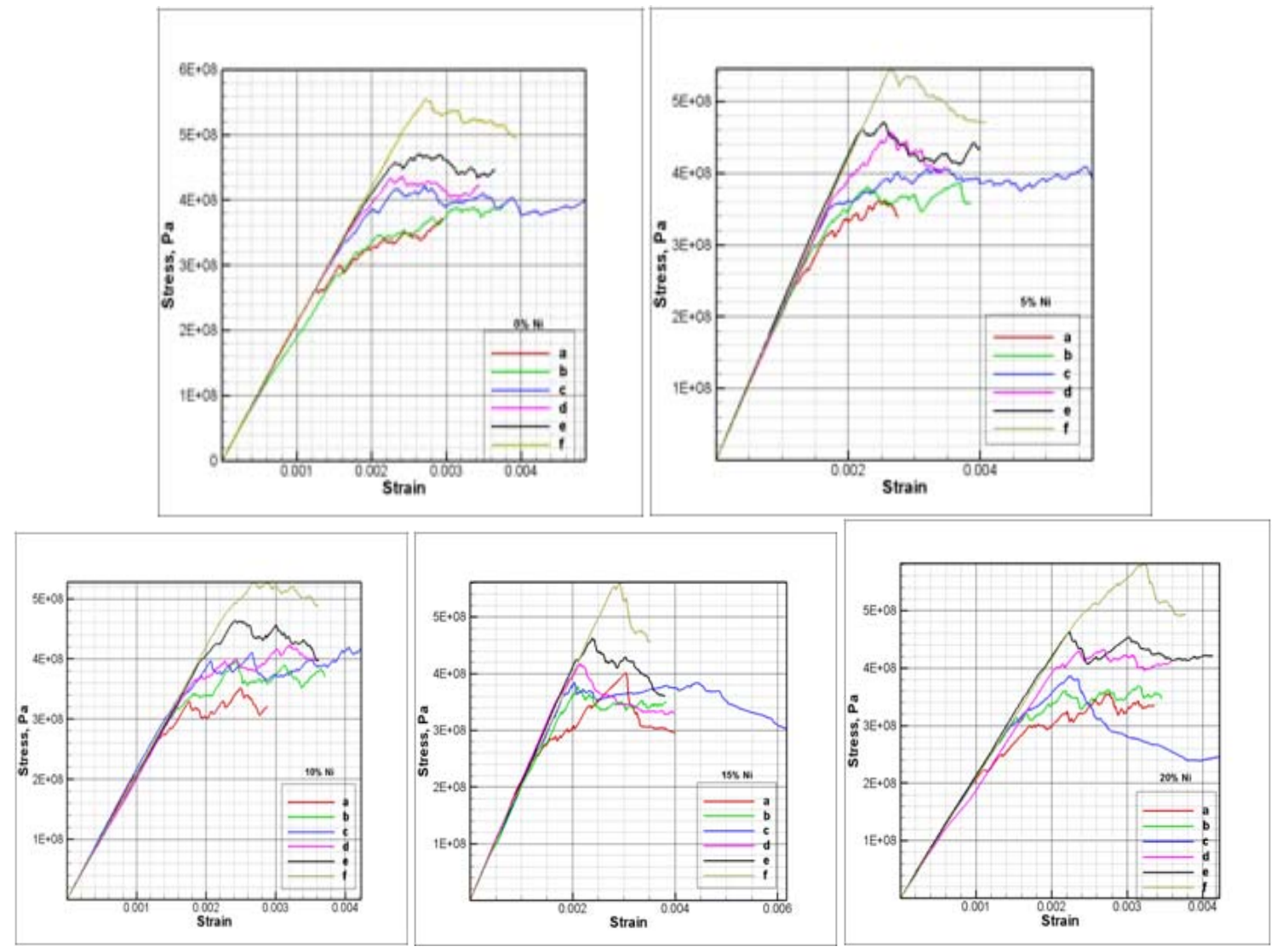

Figure 3. DD simulation results of Fe-Ni alloys: Stress-strain curves for various Ni concentrations and irradiation defect density. 

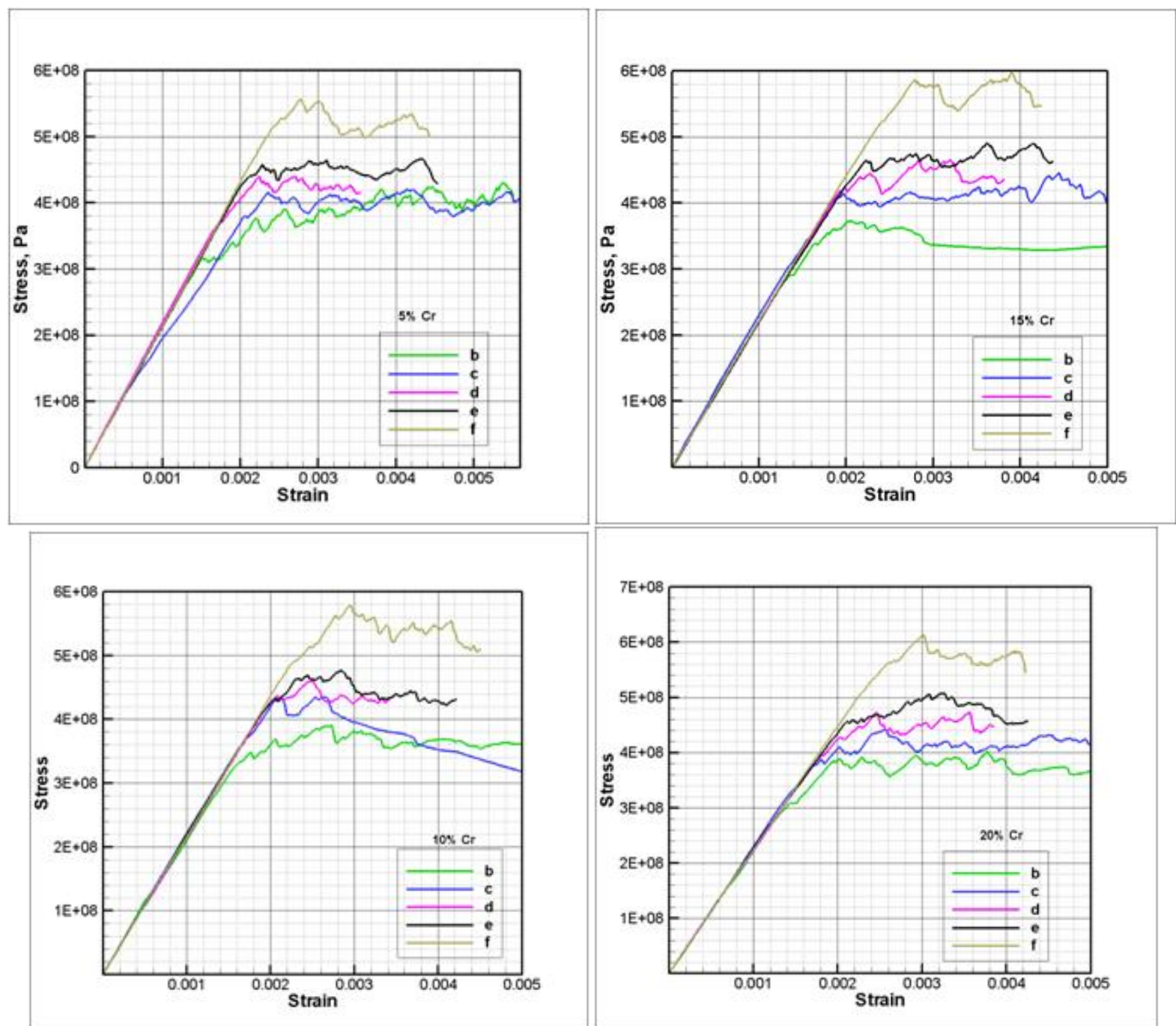

Figure 4. DD simulation results of Fe-Cr alloys: Stress-strain curves for various $\mathrm{Cr}$ concentrations and irradiation defect density.

Typical stress-strain curves predicted by the DD simulations are shown in Figures 3 and 4 for Fe-Ni and Fe-Cr systems respectively. The figures show the dependence of the stress on both $\mathrm{Ni}$ and $\mathrm{Cr}$ concentration as well as on the irradiation defect density. The figures clearly show strong dependence on the initial defect density. In all cases, the stress initially increases linearly until it is high enough for dislocations to overcome internal barriers and the pinning effect of the defects, which arises only from dislocation-FS loops elastic interactions. As the dislocations propagate and interact with the defects, their interaction energy with the defect at a critical distance can cause defect absorption within the dislocation core, which, in turn, leads to a drop in 
stress [35] and a decrease in defect density. Figure 5 shows typical DD results for the evolution of the defect density with deformation. In all cases, the defect density remains unchanged during the elastic loading as expected. As the stress becomes high enough to cause the dislocations to move, the dislocations interact with the defects and some of them get annihilated, causing a decrease in the defect density. In the same time, the dislocation densities in Fe-Ni alloys with different initial defect density generally increase with deformation, as shown in Figure 6.

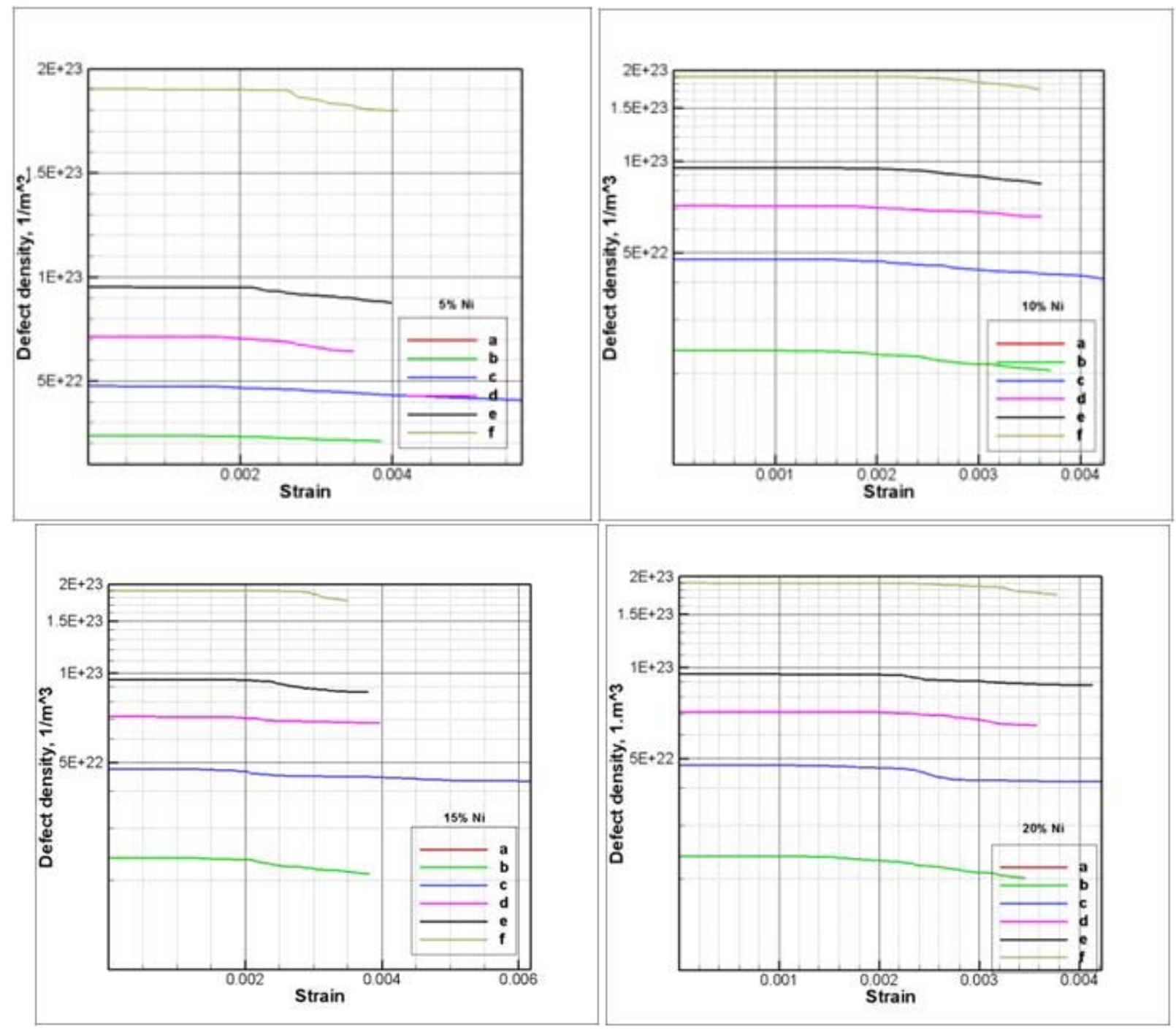

Figure 5. Fe-\%Ni DD results: Evolution of defect density for various Ni concentrations and irradiation defect density. 

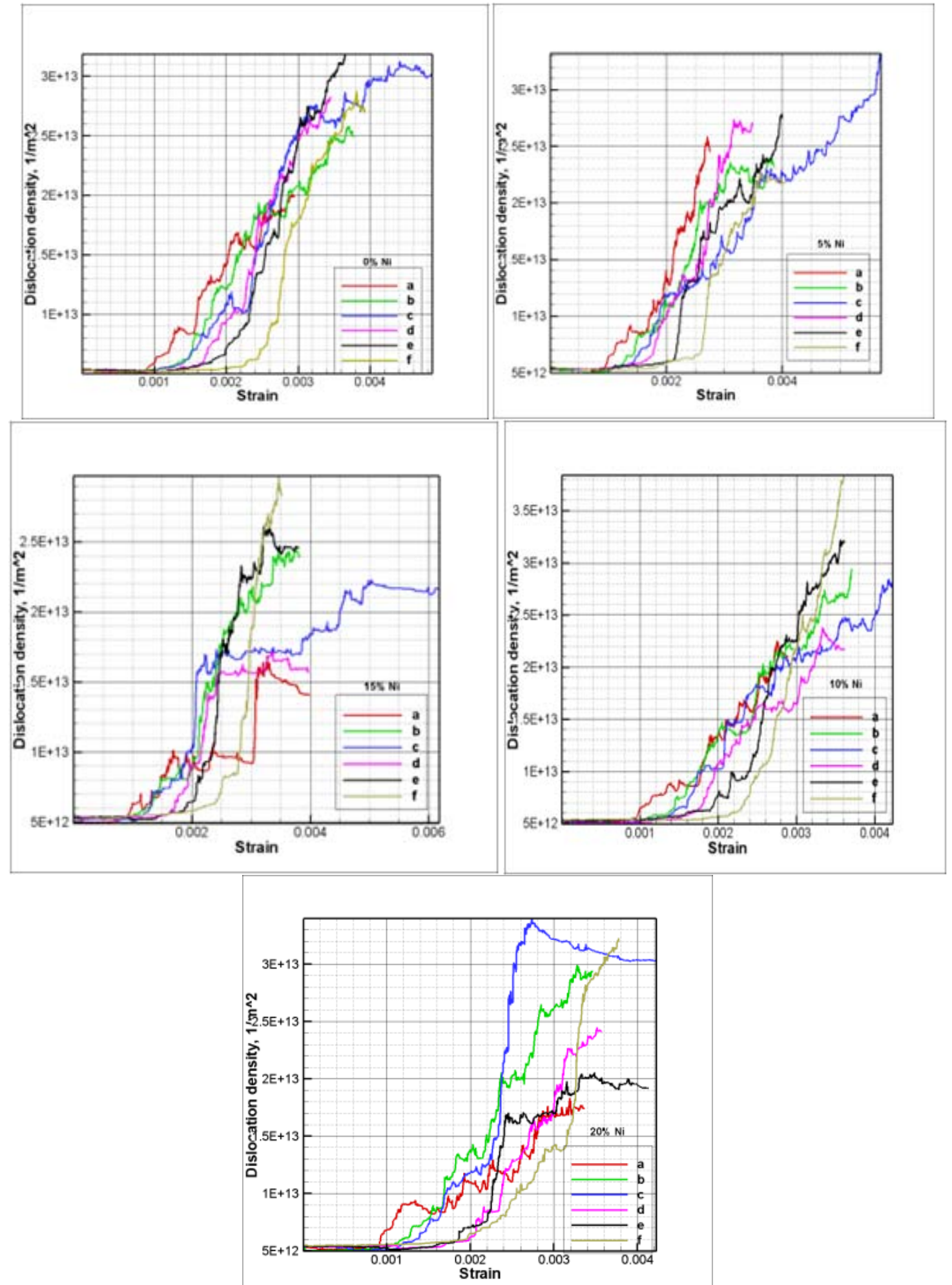

Figure 6. Fe-\%Ni DD results: Evolution of dislocation density for various Ni concentrations and irradiation defect density. 
The critical resolved shear stress (CRSS) can be extracted from the above stress strain curves. Due to the apparent fluctuations in the curves, three values are extracted, namely: the initial yield stress (first deviation form linearity), $0.02 \%$ offset yield and $0.04 \%$ offset yield. The results are summarized in Tables 4 and 5.

Table 4. DD results: CRSS in Fe-\%Ni systems

\begin{tabular}{|c|c|c|c|c|c|c|c|c|c|c|c|c|c|c|c|c|c|c|c|}
\hline & & \multicolumn{3}{|c|}{ defect density } & \multicolumn{3}{|c|}{ defect density } & \multicolumn{3}{|c|}{ defect density } & \multicolumn{3}{|c|}{ defect density } & \multicolumn{3}{|c|}{ defect density } & \multicolumn{3}{|c|}{ defect density } \\
\hline & & & a & & & $b$ & & & c & & & $d$ & & & e & & & $f$ & \\
\hline & & & 0 & & & $2 E+22$ & & & $5 E+22$ & & & $7 E+22$ & & & $1 E+23$ & & & $2 E+23$ & \\
\hline & & \multicolumn{3}{|c|}{ CRSS, Mpa } & \multicolumn{3}{|c|}{ CRSS, Mpa } & \multicolumn{3}{|c|}{ CRSS, Mpa } & \multicolumn{3}{|c|}{ CRSS, Mpa } & \multicolumn{3}{|c|}{ CRSS, Mpa } & \multicolumn{3}{|c|}{ CRSS, Mpa } \\
\hline $\mathrm{Ni} \%$ & Mobility & IY & $0.02 \%$ & $0.04 \%$ & IY & $0.02 \%$ & $0.04 \%$ & IY & $0.02 \%$ & $0.04 \%$ & IY & $0.02 \%$ & $0.04 \%$ & IY & $0.02 \%$ & $0.04 \%$ & IY & $0.02 \%$ & $0.04 \%$ \\
\hline 0 & $2.38 E+03$ & 259 & 295 & 305 & 310 & 335 & 350 & 360 & 379 & 415 & 410 & 432 & 439 & 450 & 465 & 475 & 505 & 556 & 533 \\
\hline 5 & $2.79 E+03$ & 265 & 310 & 340 & 300 & 345 & 380 & 355 & 380 & 405 & 370 & 410 & 460 & 460 & 470 & 475 & 535 & 520 & 521 \\
\hline 10 & $3.94 \mathrm{E}+03$ & 270 & 315 & 300 & 310 & 340 & 350 & 340 & 395 & 380 & 360 & 380 & 405 & 420 & 460 & 465 & 500 & 510 & 525 \\
\hline 15 & $5.00 E+03$ & 285 & 310 & 310 & 320 & 370 & 360 & 360 & 380 & 375 & 385 & 420 & 400 & 420 & 460 & 440 & 560 & 540 & 535 \\
\hline 20 & $5.18 \mathrm{E}+03$ & 300 & 301 & 320 & 310 & 340 & 360 & 320 & 350 & 382 & 400 & 425 & 435 & 460 & 440 & 420 & 510 & 560 & 582 \\
\hline
\end{tabular}

Table 5. DD: Results: CRSS in Fe-\%Cr systems

\begin{tabular}{|c|c|c|c|c|c|c|c|c|c|c|c|c|c|c|c|c|c|c|c|}
\hline & & \multicolumn{3}{|c|}{ defect density } & \multicolumn{3}{|c|}{ defect density } & \multicolumn{3}{|c|}{ defect density } & \multicolumn{3}{|c|}{ defect density } & \multicolumn{3}{|c|}{ defect density } & \multicolumn{3}{|c|}{ defect density } \\
\hline & & & a & & & $b$ & & & c & & & $d$ & & & e & & & $f$ & \\
\hline & & & 0 & & & $2 E+22$ & & & $5 \mathrm{E}+22$ & & & $7 E+22$ & & & $1 E+23$ & & & $2 E+23$ & \\
\hline & & \multicolumn{3}{|c|}{ CRSS, Mpa } & \multicolumn{3}{|c|}{ CRSS, Mpa } & \multicolumn{3}{|c|}{ CRSS, Mpa } & \multicolumn{3}{|c|}{ CRSS, Mpa } & \multicolumn{3}{|c|}{ CRSS, Mpa } & \multicolumn{3}{|c|}{ CRSS, Mpa } \\
\hline $\mathrm{Ni} \%$ & Mobility & $\mathrm{IY}$ & $0.02 \%$ & $0.04 \%$ & IY & $0.02 \%$ & $0.04 \%$ & IY & $0.02 \%$ & $0.04 \%$ & IY & $0.02 \%$ & $0.04 \%$ & IY & $0.02 \%$ & $0.04 \%$ & IY & $0.02 \%$ & $0.04 \%$ \\
\hline 0 & $2.38 \mathrm{E}+03$ & 183 & 209 & 216 & 219 & 237 & 247 & 255 & 268 & 293 & 290 & 305 & 310 & 318 & 329 & 336 & 357 & 393 & 377 \\
\hline 5 & $2.79 \mathrm{E}+03$ & 187 & 219 & 240 & 212 & 246 & 269 & 251 & 269 & 286 & 262 & 290 & 325 & 325 & 332 & 336 & 378 & 368 & 368 \\
\hline 10 & $3.94 \mathrm{E}+03$ & 191 & 223 & 212 & 219 & 240 & 248 & 240 & 279 & 269 & 255 & 269 & 286 & 297 & 325 & 329 & 354 & 361 & 371 \\
\hline 15 & $5.00 \mathrm{E}+03$ & 202 & 219 & 219 & 226 & 262 & 255 & 255 & 269 & 265 & 272 & 297 & 283 & 297 & 325 & 311 & 396 & 382 & 378 \\
\hline 20 & $5.18 \mathrm{E}+03$ & 212 & 215 & 226 & 219 & 240 & 255 & 226 & 248 & 270 & 283 & 301 & 308 & 325 & 311 & 297 & 361 & 396 & 412 \\
\hline
\end{tabular}

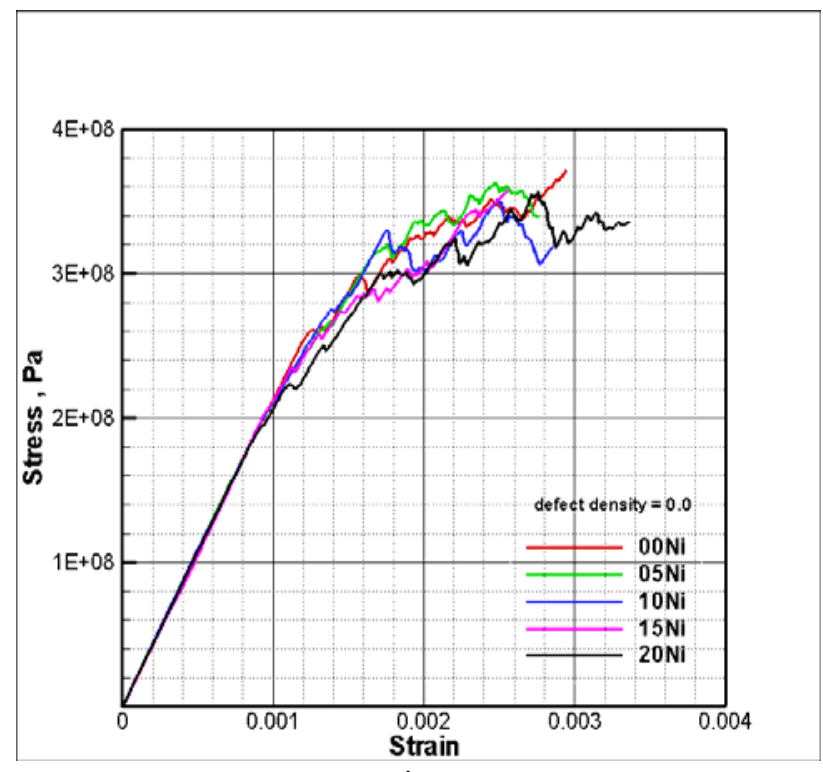

a)

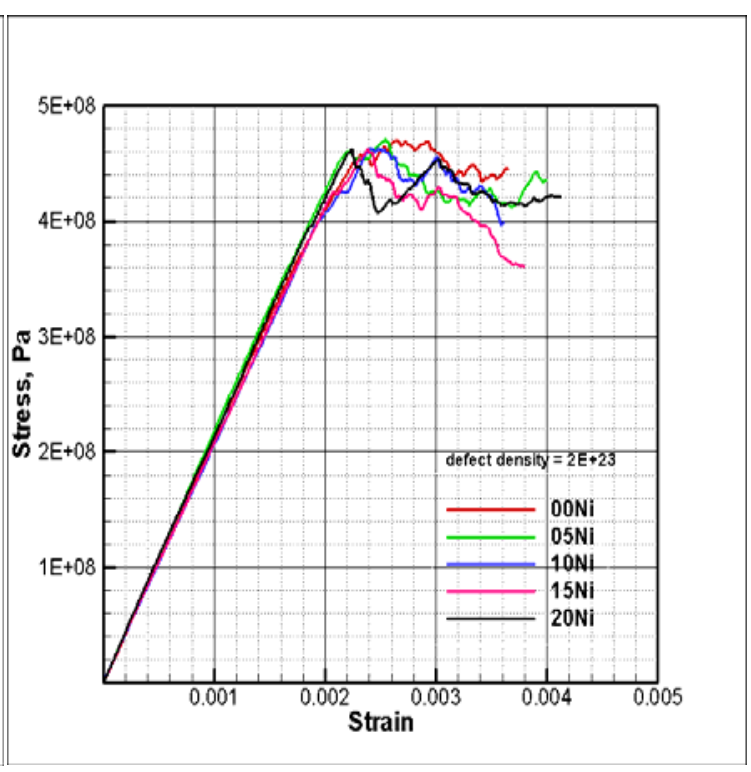

b)

Figure 7. Fe-\%Ni- Dislocation dynamics results: Effect of Ni concentrations 
Figure 7 illustrates the effect of Ni concentration on the stress-strain behavior with or without defects. Figure $7 \mathrm{a}$ depicts the effect of the concentration, therefore the dislocation mobility on the stress-strain behavior for the case with no defects, while Figure $7 \mathrm{~b}$ shows the effect when there are defects. Similar trends are observed for all defect densities for both $\mathrm{Fe}-\mathrm{Ni}$ and $\mathrm{Fe}-\mathrm{Cr}$ systems.

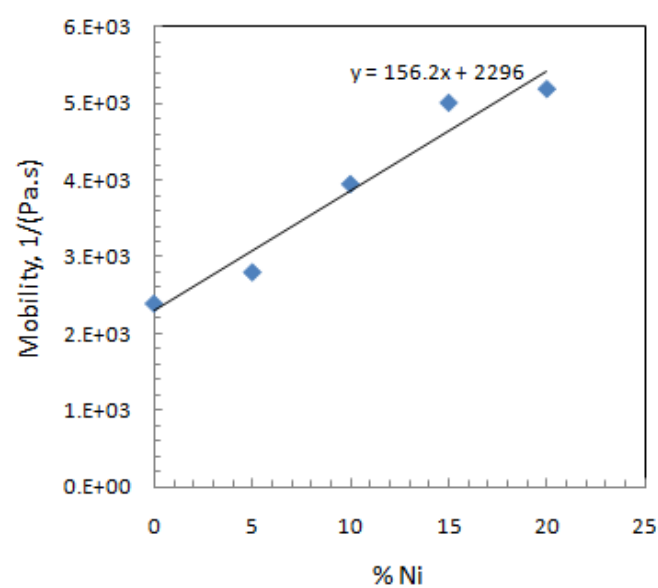

a)

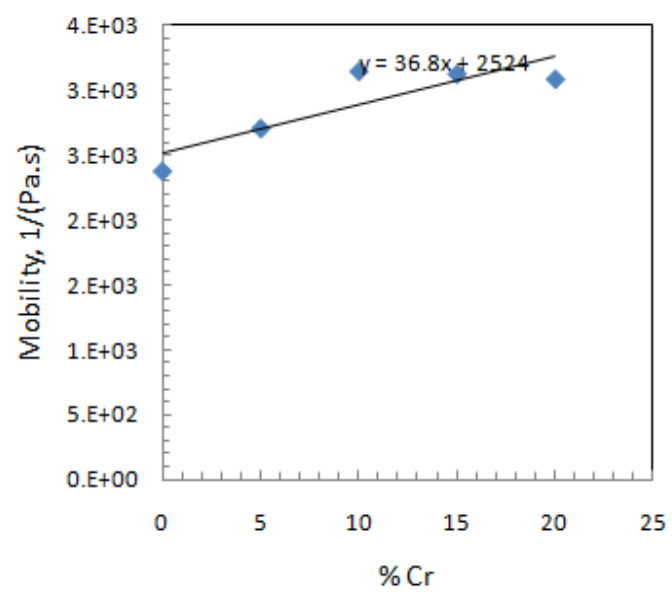

c)

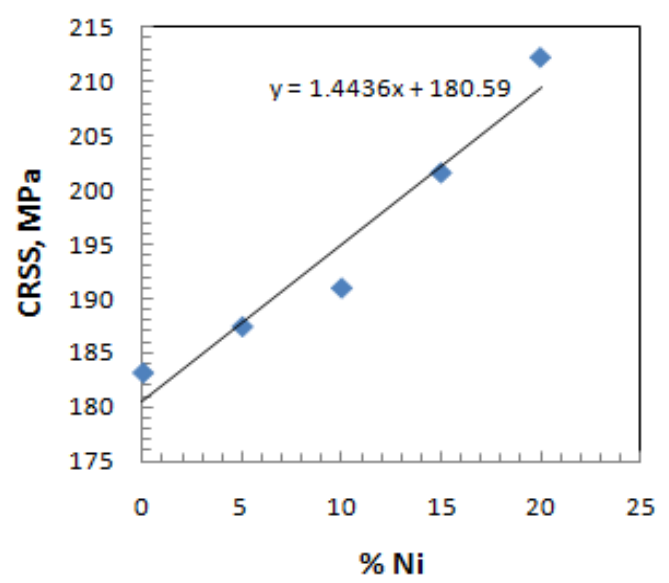

b)

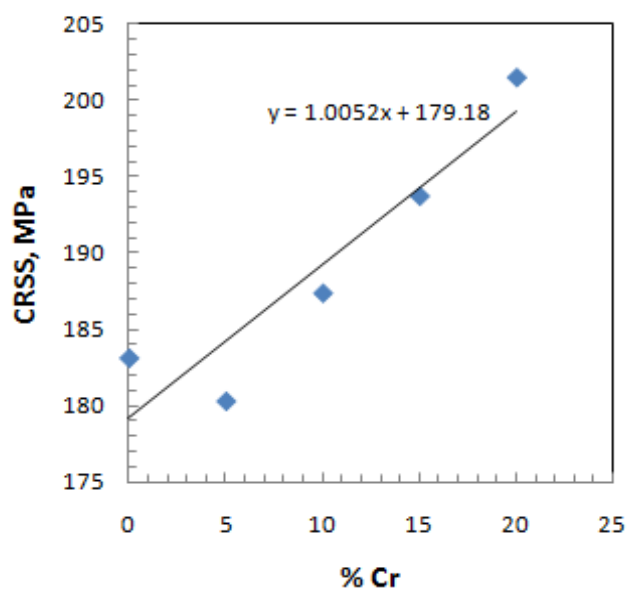

d)

Figure 8. The dislocation mobility and CRSS for Fe-NI and Fe-Cr systems with no defects. 
This effect is further shown in Figures 8a-d for the case of no defects. These results show that the CRSS depends linearly on the concentration. This effect is most pronounced when the defect density is low because the magnitude of the stress resulting from the irradiation hardening becomes much larger than the increase due to the concentration as the defect density increases. The increase in the CRSS as a function of defect density $\rho_{D}$ is shown in Figures 9 and 10.
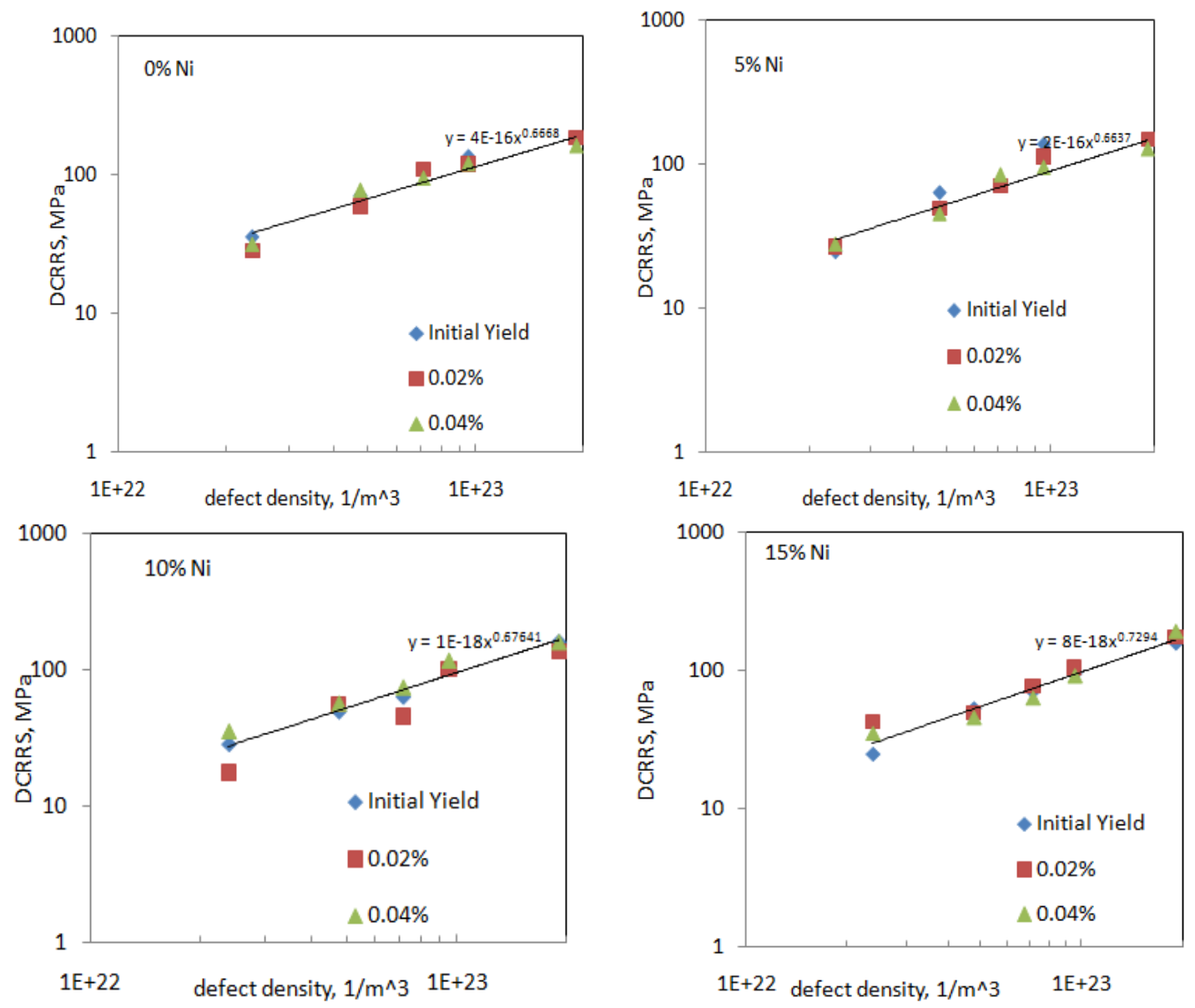


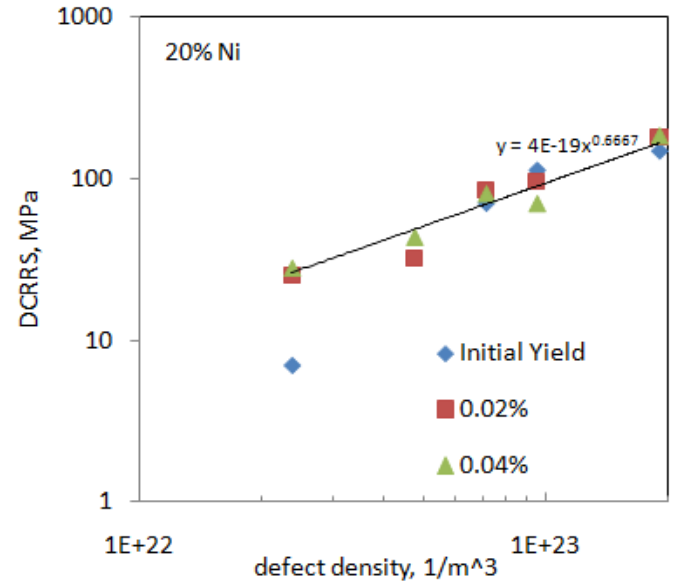

Figure 9. Increase of the CRSS with irradiation defect density in Fe-Ni.
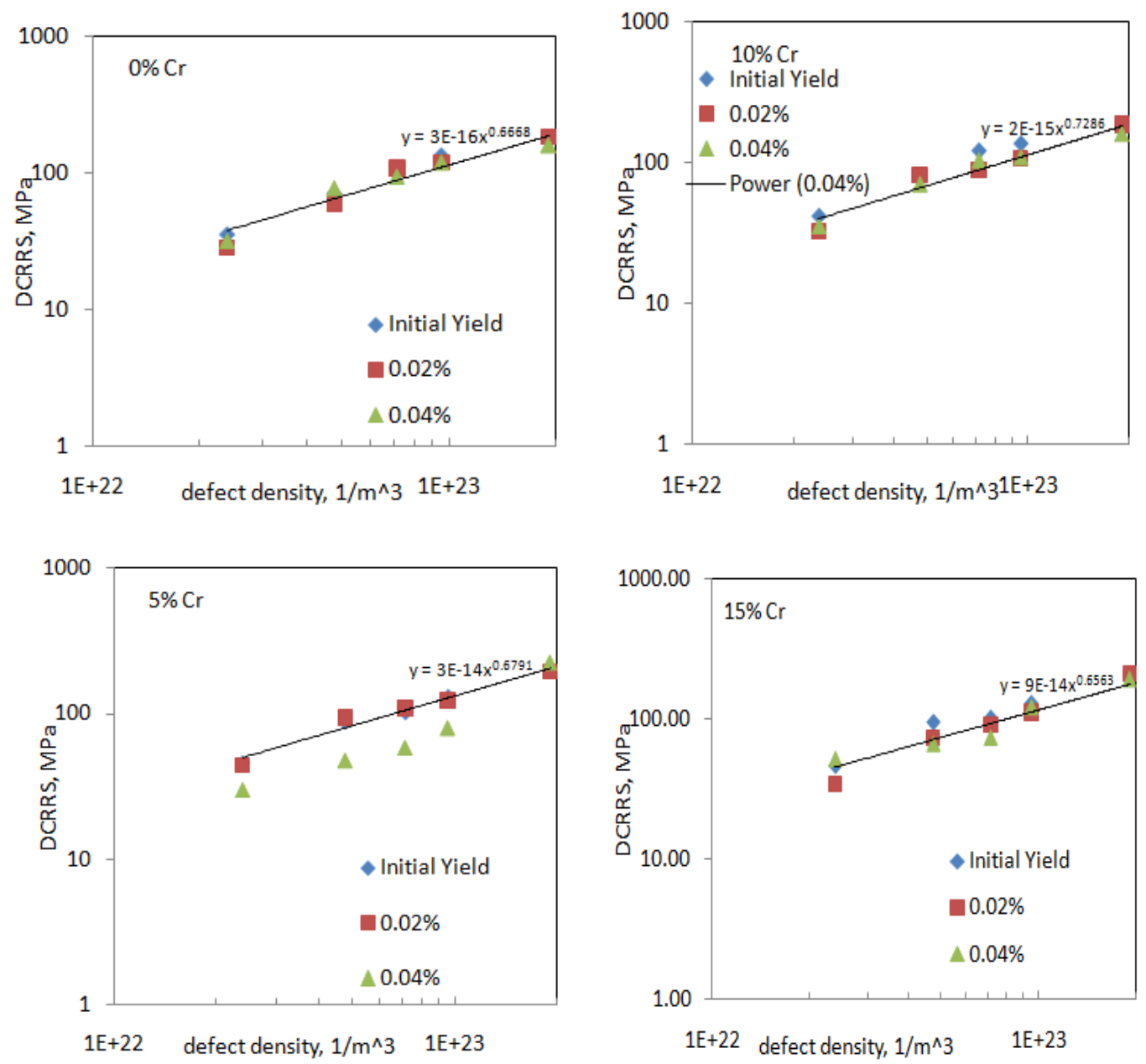


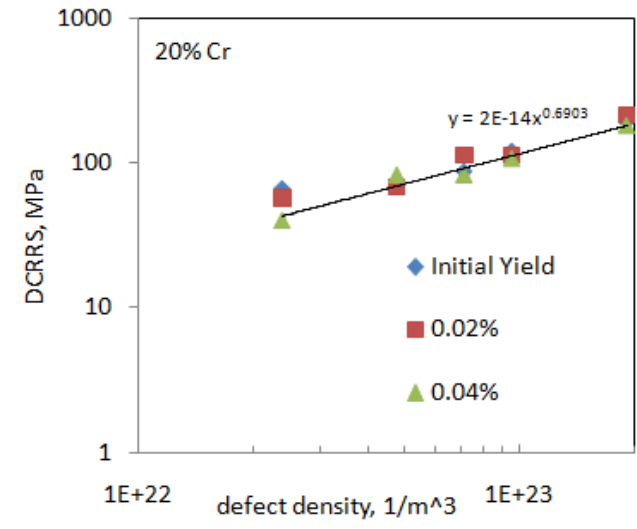

Figure 10. Increase of the CRSS with irradiation defect density in $\mathrm{Fe}-\mathrm{Cr}$.

The increase DCRSS $(=\Delta \tau)$ is the value of the CRSS $\left(=\tau\left(\rho_{\boldsymbol{D}}, \boldsymbol{c}\right)\right)$ for a given mobility/concentration $(c)$ and for a given defect density minus the reference state with no defects, $\tau_{0}(\boldsymbol{c})$ :

$$
\Delta \tau=\tau\left(\rho_{D}, c\right)-\tau_{0}(c)
$$

It can be deduced from Figures 9 and 10 that the increase in the CRSS follows a similar trend for all the Fe-Cr and Fe-Ni cases studied. In all the cases the curves can be fitted to a simple power in $\rho_{\boldsymbol{D}}$ and linear dependence on $c$, leading to the following constitutive law for the CRSS.

$$
\tau\left(\rho_{D}, c\right)=\tau_{0}+\tau_{c} c+\alpha \mu(c)\left(b^{2} d \rho_{D}\right)^{n}
$$

where $\tau_{0}$ is the yield stress for the case with no defects and zero concentration, i.e. pure $\alpha-F e$; $\tau_{c}$ is a constant equal to approximately $144 \mathrm{MPa}$ for $\mathrm{Ni}$ and $100 \mathrm{MPa}$ for $\mathrm{Cr}$ (see Figure 8); $\alpha$ is a constant on the order of $0.1-0.3 ; b$ is the magnitude of the Burgers vector; $d$ is the average size of the defect (1-3 nm); and $n$ is a constant with an average value ranging between 0.4 and 0.6. This relationship is different from the dispersed barrier model [62-64], which is based on geometrical considerations for strong obstacles intersecting the dislocation glide planes, where the increase in yield strength is assumed to be proportional to the square root of the defect cluster density [65-66]. However, in DD the pinning effect is caused solely from the elastic interaction between the glide dislocations and the FS loops which is an explicit representation of the interaction process. This effect is similar to that discussed in [67] where a model was developed to account for the elastic interaction between dislocation loops situated near the dislocation core, 
leading to a modification of the dispersed hardening model. As noted earlier, in the dispersed hardening mode it is assumed that glide dislocations interact with strong obstacles in a glide plane and the yield stress $\tau$ is inversely proportional to $\ell$, the average spacing between obstacles. In the case of dislocation-dislocation interaction (forest hardening), the spacing between forest dislocations (obstacles) is equal to $1 / \sqrt{\rho}$, where, in this case, $\rho$ is the dislocation density defined as the total dislocation length per unit volume, leading to $\tau \propto \mu b \sqrt{\rho}$. In the case of hardening induced from dislocation interaction with cluster obstacles, such as FS loops of average size $d$ whose density is defined as the number of defects per unit volume and to maintain the form of the dispersed hardening model, the average obstacle spacing is assumed to be $1 / \sqrt{d \rho_{D}}$, leading to $\tau \propto \mu b \sqrt{d \rho_{\boldsymbol{D}}}$. However, in a more precise treatment the average spacing between obstacles dispersed in a three-dimensional space is equal to $1 / \sqrt[3]{\rho_{D}}$. Thus, Equation (3) can be written as

$$
\tau\left(\rho_{D}, c\right)=\tau_{0}+\tau_{c} c+\alpha \mu(c)\left(\frac{b^{2} d}{\ell^{3}}\right)^{n}, \ell / \sqrt[3]{\rho_{D}}
$$

This equation provides a generalized from for the dependence of the yield strength on concentration, and irradiation defect density.

As a result of dislocation-defect interaction, the defect density $\rho_{D}$ decreases during plastic deformation (as can be deduced from Figure 5). As previously mentioned, as the dislocations begin to move at the CRSS, they interact with the defects and some get annihilated, causing a decrease in the defect density. Because the dislocations are stationary, no defects get annihilated during elastic loading. Therefore, the evolution of defect density should be presented in terms of the plastic strain. A typical defect density versus plastic stain is shown in Figure 11 for one case. All other cases follow the same trends. Based on these results, a generalized evolution law for the defect density can be derived.

Consistent with the discrete DD of dislocation-obstacle interaction, some of these defects get annihilated as a glide dislocation passes through a cloud of the FS defects (depending on their interaction energy with the glide dislocation [36]). Let $N_{d}$ be the number of defects in volume $\mathrm{V}$ centered around the dislocation segment, then $N_{d}=V \rho_{D}$. Only a fraction $\beta \leq 1$ of these defects 
may be annihilated. Their annihilation rate depends on the average time $t$ for one dislocation with velocity $v_{D}{ }^{*}$ to move through the volume, which equals $t=v_{D} / R^{*}$, where $R^{*}$ is the average distance traveled by a dislocation through the volume $V$. If the volume is assumed to be spherical with radius $R$, then $R^{*}=\pi R / 4$. The frequency $f$ with which irradiation defects within the volume $V$ get annihilated is equal to $f=\beta N_{d} / t$. Given that $N$ glide dislocations may go through the volume $V$, the rate of decrease of the defect density within $V$ becomes:

$$
\dot{\rho}_{D}=-\frac{f}{V} N
$$

Noting the number of dislocations crossing the volume $V$ is equal to $N=\rho_{d} A$, where $\rho_{d}$ is the dislocation density and $A$ is the project area of $V$, assuming a spherical volume of radius $R$, and after a few algebraic manipulations Equation (5) becomes:

$$
\dot{\rho}_{\boldsymbol{D}}=-4 \beta R \rho_{D} \rho_{d} v_{D}
$$

This expression provides an evolution law for FS defects in bcc materials systems, resulting from the absorption of FS loops by gliding dislocations. In the case of fcc materials systems, such as $\mathrm{Cu}$, where the dominate defect is dislocation stacking fault tetrahedral (SFT) interaction, the evolution of the defect density involves the mechanism of dislocation cutting of the initially perfect SFT as discussed in $[38,68]$.

By using the Orowan relation $\dot{\gamma}^{p}=\rho_{d} b v_{D}$, equation (6) can be written as:

$$
\dot{\rho}_{D}=-4 \beta \frac{R}{b} \rho_{D} \dot{\gamma}^{p}
$$

Assuming $\beta$ is constant (in a more general treatment, it may depend on both the defect density and dislocation density), equation (7) can be integrated to yield the analytical solution:

$$
\rho_{\boldsymbol{D}}=\rho_{\boldsymbol{D}}^{0} \boldsymbol{e}^{-4 \beta\left(\frac{R}{b}\right) \gamma^{p}},
$$

where $\rho_{D}^{0}$ is the initial defect density. Equation (8) can be fitted to the results from the discrete DD simulations, and typical results are shown in Figure 11, showcasing almost a perfect fit of 
the DD results to the model with the exponential coefficient $4 \beta R / b$ varying between 0.9 to 1.0 , depending on the initial defect density.

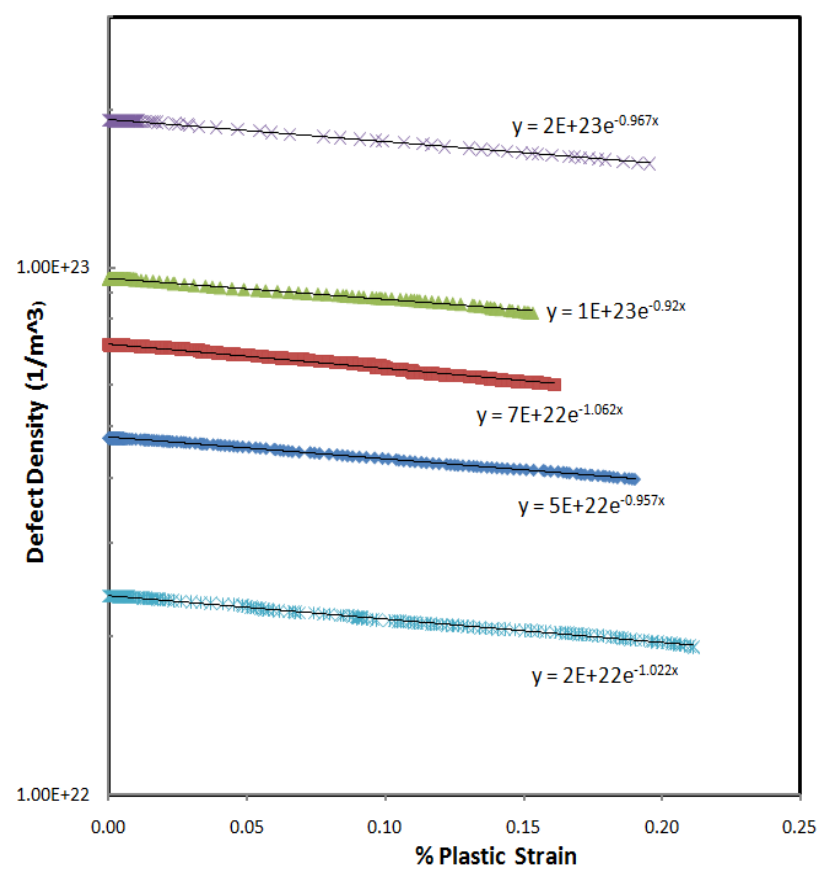

Figure 11. Evolution of defect density as a function plastic strain for $\mathrm{Fe}-10 \% \mathrm{Ni}$.

Based on results from DD analyses, a constitutive equation for the irradiation-induced hardening in $\mathrm{Fe}-\mathrm{Cr}$ and $\mathrm{Fe}-\mathrm{Ni}$ systems and its dependence on alloying element concentration are derived, primarily in equations (4) and (7). These DD-based equations, along with other DD results such as evolution of DD (Figure 6), junctions, jogs, and cross slip, form the basis for the continuum-DD-based crystal plasticity under development.

\subsection{Continuum Dislocation Dynamics - Crystal Plasticity}

Dislocation density evolution and the mobile-dislocation-related CRSS are predicted from discrete DD. They are the basis for the continuum-DD-based crystal plasticity. Here, we present the framework of continuum DD applied in steel. This framework used the principle of crystal plasticity and was improved by introducing more physical-based mechanisms to substitute empirically based formulations on constitutive and hardening laws. In crystal plasticity [69-70], the total deformation gradient $F$ is the product of elastic part $F^{e}$ and plastic part $F^{p}$ : 


$$
F=F^{e} F^{p}
$$

where $F^{e}$ is due to the elastic distortion of lattice and $F^{p}$ is due to the slip by the dislocation motion in the unrotated intermediate configuration.

The plastic deformation gradient is expressed by the flow rule:

$$
\dot{F}^{p}=L^{p} F^{p}
$$

with the plastic velocity gradient expressed as the sum of all crystallographic slip rates, $\dot{\gamma}^{\alpha}$ :

$$
L^{p}=\sum_{\alpha=1}^{N} \dot{\gamma}^{\alpha} n^{\alpha} \otimes m^{\alpha}
$$

where $n^{\alpha}$ and $m^{\alpha}$ are the vectors representing slip direction and slip plane normal of slip system $\alpha$, and $\mathrm{N}$ is the number of slip systems.

The plastic velocity gradient is composed of a symmetric part, strain rate $D^{P}$, and an antisymmetric part, total spin $W^{P}$ :

$$
\begin{aligned}
& D_{i j}^{p}=\operatorname{symm}\left(L_{i j}^{p}\right)=\frac{1}{2} \sum_{\alpha=1}^{N} \dot{\gamma}^{\alpha}\left(n_{i}^{\alpha} m_{j}^{\alpha}+n_{j}^{\alpha} m_{i}^{\alpha}\right) \text { and } \\
& W_{i j}^{p}=\operatorname{symm}\left(L_{i j}^{p}\right)=\frac{1}{2} \sum_{\alpha=1}^{N} \dot{\gamma}^{\alpha}\left(n_{i}^{\alpha} m_{j}^{\alpha}-n_{j}^{\alpha} m_{i}^{\alpha}\right) .
\end{aligned}
$$

Here, strain rate $D^{P}$ determines the deformation behavior by updating stress strain curve; while total spin $W^{P}$ is used to update the orientation of crystals, further texture in polycrystalline materials.

With traditional crystal plasticity $\dot{\gamma}^{\alpha}$, the shear strain rate of each slip system $\alpha$ is determined by a strain-rate-dependent power law function:

$$
\dot{\gamma}^{\alpha}=\dot{\gamma}_{0}\left|\frac{\tau^{\alpha}}{\tau_{0}^{\alpha}}\right|^{1 / m} \operatorname{sign}\left(\tau^{\alpha}\right)
$$


where $\dot{\gamma}_{0}$ is reference strain rate, $\tau_{0}^{\alpha}$ is the slip resistance of the slip system $\alpha, \tau^{\alpha}$ is the resolved shear stress along slip system $\alpha$, and $\mathrm{m}$ is the rate sensitivity exponent. The parameters $\tau_{0}^{\alpha}$ and $\mathrm{m}$ are obtained from experimental results using curve fitting.

In contrast, continuum-DD-based crystal plasticity proposed another form of power law by using the results from discrete DD to substitute the parameters $m$ from curve fitting. Our constitutive law for the shear strain rate is based on the Orowan relation [71]:

$$
\dot{\gamma}^{\alpha}=\rho_{M}^{\alpha} b v_{g}^{\alpha}
$$

where $\rho_{M}^{\alpha}$ is the mobile dislocation density of slip system $\alpha, \mathrm{b}$ is the Burgers vector, and $v_{g}^{\alpha}$ is the average dislocation glide velocity in slip system $\alpha$.

In previously developed models, the dislocation glide velocity $v_{g}^{\alpha}$, is expressed in a power law of resolved shear stress $\tau^{\alpha}$ similar with shear strain rate of slip system $\alpha,{ }^{\tau_{0}^{\alpha}}$ :

$$
v_{g}^{\alpha}=v_{0}\left|\frac{\tau^{\alpha}}{\tau_{0}^{\alpha}}\right|^{1 / m} \operatorname{sign}\left(\tau^{\alpha}\right)
$$

Here the expression of dislocation glide velocity is improved to a general law using Kock-type activation enthalpy:

$$
v_{g}^{\alpha}= \begin{cases}v_{D} l_{g} \exp \left[-\frac{\Delta \mathrm{F}_{\mathrm{k}}}{\mathrm{kT}}\left(1-\left(\frac{\left|\tau^{\alpha}\right|-\tau_{0}^{\alpha}}{\tau_{\mathrm{P}}^{\alpha}}\right)^{\mathrm{p}}\right)^{\mathrm{q}}\right] \operatorname{sign}\left(\tau^{\alpha}\right) & \text { when }\left|\tau^{\alpha}\right|>\tau_{0}^{\alpha} \\ 0 & \text { when }\left|\tau^{\alpha}\right| \leq \tau_{0}^{\alpha}\end{cases}
$$

where $l_{g}$ is the distance between the barrier. For a kink height, $l_{g}$ is close to Burgers vector. $V_{D}$ is the Debye frequency, and $k$ is the Boltzmann constant. $T$ is temperature, $\Delta \mathrm{F}_{\mathrm{k}}$ is Kock-type activation enthalpy, $\tau_{\mathrm{P}}^{\alpha}$ is the Peierls lattice resistance of slip system $\alpha$, and $p$ and $q$ are strain rate parameters. In this new proposed law, all parameters have physical meaning calculated from lower length scale models. The slip resistance of slip system $\alpha, \tau_{0}^{\alpha}$, is a summation of reference resistance $\tau_{0}$ from lattice friction stress for moving dislocations; resistance due to dislocationdefect interaction $\tau_{d}^{\alpha}$, mainly from irradiation; and resistance from dislocation hardening $\tau_{d h}^{\alpha}$ : 


$$
\tau_{0}^{\alpha}=\tau_{0}+\tau_{d}^{\alpha}+\tau_{d h}^{\alpha}
$$

According to the Baily-Hirsch model [72], the resistance from dislocation hardening is slip resistance of statistically stored dislocations on other slip systems against moving ones on one specific slip system $\alpha$. It is a function of interaction matrix of slip systems $\Omega^{\alpha m}$ :

$$
\tau_{d h}^{\alpha}=\sum_{m} \alpha b \mu \Omega^{\alpha m} \sqrt{\rho^{m}}
$$

Here, $b$ is Burgers vector magnitude, $\mu$ is shear modulus, $\alpha$ is a numerical factor on the order of 0.1 , and $\rho^{m}$ is the density of statistically stored dislocations accumulated on the slip system $\mathrm{m}$.

The evolution rate of statistically stored dislocation density is related to the mean free path of moving dislocations on slip system $\alpha, L^{\alpha}[73]$ :

$$
\dot{\rho}^{\alpha}=c \dot{\gamma}^{\alpha} / b L^{\alpha}
$$

where $\dot{\gamma}^{\alpha}$ is shear strain rate of slip system $\alpha$ and $c$ is a numerical constant of order $1 . L^{\alpha}$ is a function of dislocation density.

If we assume defects are distributed randomly for all slip systems, $\tau_{d}^{\alpha}$ is a constant. A modified dispersed barrier hardening model is used to express irradiation resistance $\tau_{d}^{\alpha}$ from dislocation-defect interaction:

$$
\tau_{d}^{\alpha}=\beta d \mu\left(\rho_{d} d\right)^{n}
$$

where $\rho_{d}$ is the defect density and $d$ is the defect size. In a traditional dispersed barrier hardening model, $n=0.5$. In our proposed model, $n$ is calculated directly from discrete DD.

In the previously proposed DD-based crystal plasticity models, the dislocation density evolution laws are either ignored or used a fitted curve to represent the evolution (as proposed by Kock [74]):

$$
\dot{\rho}^{\alpha}=\frac{1}{b}\left(\frac{\sqrt{\sum_{\beta}^{n} \rho^{\beta}}}{k_{a}}-k_{b} \rho^{\alpha}\right)\left|\dot{\gamma}^{\alpha}\right|
$$


where $k_{a}$ and $k_{b}$ are material parameters for dislocation generation and annihilation, respectively.

In this work, the evolution rate of the dislocation density is mechanics based and calculated from the discrete DD. Generally, the dislocations are divided into two types, mobile and immobile:

$$
\rho^{\alpha}=\rho_{M}^{\alpha}+\rho_{I}^{\alpha} .
$$

The evolution of mobile dislocation density is composed of six terms with different physical contribution. The first mechanism is from the multiplication and growth of residential dislocation and the production of new dislocations from Frank-Reed sources in slip system $\alpha$ :

$$
\dot{\rho}_{M}^{\alpha}=\alpha_{1} \rho_{M}^{\alpha} \frac{\bar{v}_{g}^{\alpha}}{\bar{l}^{\alpha}},
$$

where $\alpha_{1}$ is the dislocation multiplication coefficient, $\rho_{M}^{\alpha}$ is the mobile dislocation density distributed on slip system $\alpha$, and $\bar{l}^{\alpha}$ is the mean free path of dislocations on slip system $\alpha$.

The second mechanism captures the mutual annihilation of two mobile edge or screw dislocations with opposite signs in slip system $\alpha$ :

$$
\dot{\rho}_{M}^{\alpha}=-2 \alpha_{2} R_{c}\left(\rho_{M}^{\alpha}\right)^{2} \bar{v}_{g},
$$

where $\alpha_{2}$ is the dislocation annihilation coefficient and $R_{c}$ is the capture radius for the dislocation annihilation event.

The third mechanism describes the transition of mobile type to immobile type due to the interaction between dislocations:

$$
\dot{\rho}_{M}^{\alpha}=-\alpha_{3} \rho_{M}^{\alpha} \frac{\bar{v}_{g}}{\bar{l}},
$$

where $\alpha_{3}$ is the immobilization parameter.

Conversely, the fourth mechanism is about the mobilization of immobile dislocations due to breaks of junctions, dipoles, pinning parts, etc., at a critical stress condition: 


$$
\dot{\rho}_{M}^{\alpha}=\alpha_{4}\left(\frac{\left|\tau^{\alpha}\right|}{\tau^{*}}\right)^{r} \rho_{I}^{\alpha} \frac{\bar{v}_{g}^{\alpha}}{\bar{l}}
$$

where $\alpha_{4}$ is the mobilization parameter.

The fifth mechanism considers cross slip, the phenomena where screw dislocation segment on one slip plane to move to another plane. This mechanism was not evaluated in most models.

$$
\dot{\rho}_{M}^{\alpha}=\alpha_{5} \sum_{\beta=1}^{N} P^{\alpha \beta} \rho_{M}^{\beta} \frac{\bar{v}_{g}^{\alpha}}{\bar{l}}
$$

where $\alpha_{5}$ is the cross slip parameter and $P^{\alpha \beta}$ is a delta function matrix based on the stochastic process to determine of the probability of cross slip.

The sixth mechanism is similar with the second one, also an annihilation, but between mobile and immobile type dislocations:

$$
\dot{\rho}_{M}^{\alpha}=-\alpha_{6} R_{c} \rho_{I}^{\alpha} \rho_{M}^{\alpha} \bar{v}_{g}^{\alpha}
$$

Combining all of the preceding considerations, the evolution rate of mobile dislocations is:

$$
\dot{\rho}_{M}^{\alpha}=\alpha_{1} \rho_{M}^{\alpha} \frac{\bar{v}_{g}^{\alpha}}{\bar{l}}-2 \alpha_{2} R_{c}\left(\rho_{M}^{\alpha}\right)^{2} \bar{v}_{g}-\alpha_{3} \rho_{M}^{\alpha} \frac{\bar{v}_{g}}{\bar{l}}+\alpha_{4}\left(\frac{\left|\tau^{\alpha}\right|}{\tau^{*}}\right)^{r} \rho_{I}^{\alpha} \frac{\bar{v}_{g}^{\alpha}}{\bar{l}}+\alpha_{5} \sum_{\beta}^{N} p^{\alpha \beta} \rho_{M}^{\beta} \frac{\bar{v}_{g}^{\alpha}}{\bar{l}}-\alpha_{6} R_{c} \rho_{I}^{\alpha} \rho_{M}^{\alpha} \bar{v}_{g}^{\alpha}
$$

The aforementioned mechanisms of mobile dislocations evolution, including Eq. (26), (27), and (29), also involve the immobile dislocations. Based on these, the evolution rate of immobile dislocation density is:

$$
\dot{\rho}_{I}^{\alpha}=\alpha_{3} \rho_{M}^{\alpha} \frac{\bar{v}_{g}^{\alpha}}{\bar{l}}-\alpha_{4}\left(\frac{\left|\tau^{\alpha}\right|}{\tau^{*}}\right)^{r} \rho_{I} \frac{\bar{v}_{g}^{\alpha}}{\bar{l}}-\alpha_{6} R_{c} \rho_{I}^{\alpha} \rho_{M}^{\alpha} \bar{v}_{g}^{\alpha}
$$

The advantage of this framework is the physical meaning in the mesoscale model. With consideration of cross slip, anisotropic Peierls stress for different slip systems and the continuum DD will predict the strength and deformation behavior of single crystal with more fidelity. 
This model was used to predict the deformation behavior of single crystal $\alpha$-Fe. The parameters used are listed in Table 6, partly built from Lee's previous works [75-76].

Table 6. List of parameters used in the continuum dislocation dynamics model

\begin{tabular}{|l|l|l|}
\hline Symbol & Denotation & Value \\
\hline$\mu$ & Shear modulus & $80 \mathrm{GPa}$ \\
\hline$v$ & Poisson's ratio & 0.3 \\
\hline$C_{11}$ & Anisotropic elasticity constant & $242 \mathrm{GPa}$ \\
\hline$C_{12}$ & Anisotropic elasticity constant & $150 \mathrm{GPa}$ \\
\hline$C_{44}$ & Anisotropic elasticity constant & $112 \mathrm{GPa}$ \\
\hline$\gamma_{0}$ & Reference strain rate & $4 \times 10^{-5} \mathrm{~m} / \mathrm{s}$ \\
\hline$m$ & Strain rate sensitivity & 0.012 \\
\hline$\alpha$ & Baily-Hirsch hardening coefficient & 0.4 \\
\hline$b$ & Magnitude of Burger vector & $2.54 \times 10^{-10} \mathrm{~m}$ \\
\hline$\tau_{0}$ & Peierls stress (internal friction) & $11.0 \mathrm{MPa}$ \\
\hline$\beta$ & Irradiation hardening coefficient & 0 \\
\hline$\alpha_{1}$ & Mobile dislocation multiplication coefficient & 0.02 \\
\hline$\alpha_{2}$ & Mobile-mobile dislocation annihilation coefficient & 1.0 \\
\hline$R_{c}$ & Critical radius for annihilation in units of Burger vector & 15 \\
\hline$\alpha_{3}$ & Immobilization parameter, mobile to immobile & 0.002 \\
\hline$\alpha_{4}$ & mobilization parameter, Mobile to immobile & 0.002 \\
\hline$\alpha_{5}$ & Cross slip coefficient & 0.016 \\
\hline$\alpha_{6}$ & Mobile-immobile annihilation coefficient & 1.0 \\
\hline
\end{tabular}

One point should be emphasized here: the parameters are either experimental measured (such as $\mu$ and $C_{11}$ ) or calculated from the discrete DD (e.g., the parameters in the mechanisms of dislocation density evolution law, $\alpha_{1}, \alpha_{2}$, etc.). Along with the advantages already mentioned, there are four other bright points in the proposed framework. The first is the application of cross slips, the second is the anisotropic Peierls stress, the third is the updated strain rate sensitivity law with information of dislocation density, and the final one is the capability to predict irradiation hardening by introducing hardening due to interaction between dislocation and 
defects. The predicted stress strain curve of single crystal $\alpha$-Fe with the uniaxial tension loading direction along the crystal orientation $<001>$ is illustrated in Figure 12. Compared with the experimental results measured by Keh et al. [77], the predicted results are in good agreement with the experimental results.

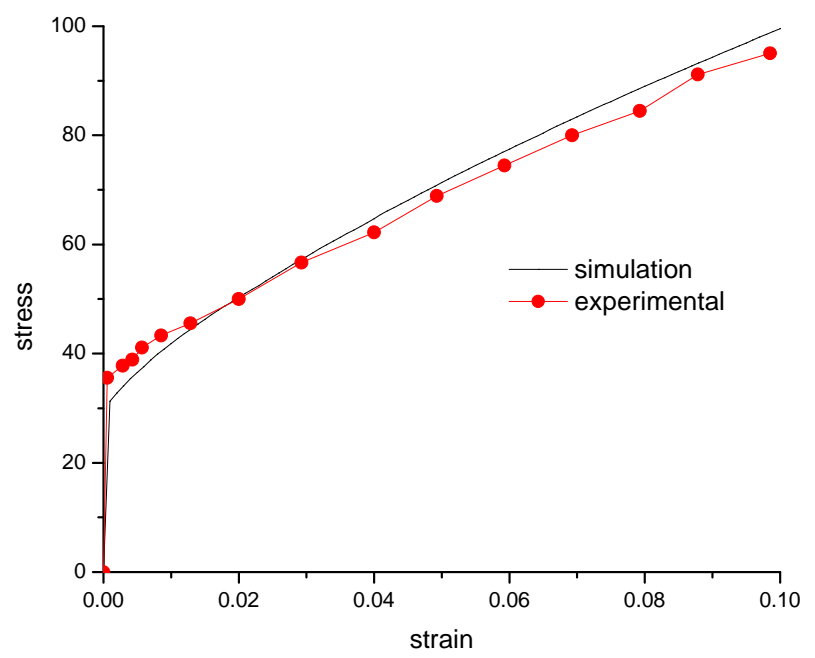

Figure 12. Measured and predicted stress strain curve of single crystal $\alpha$-Fe under uniaxial tension along $<001>$ direction.

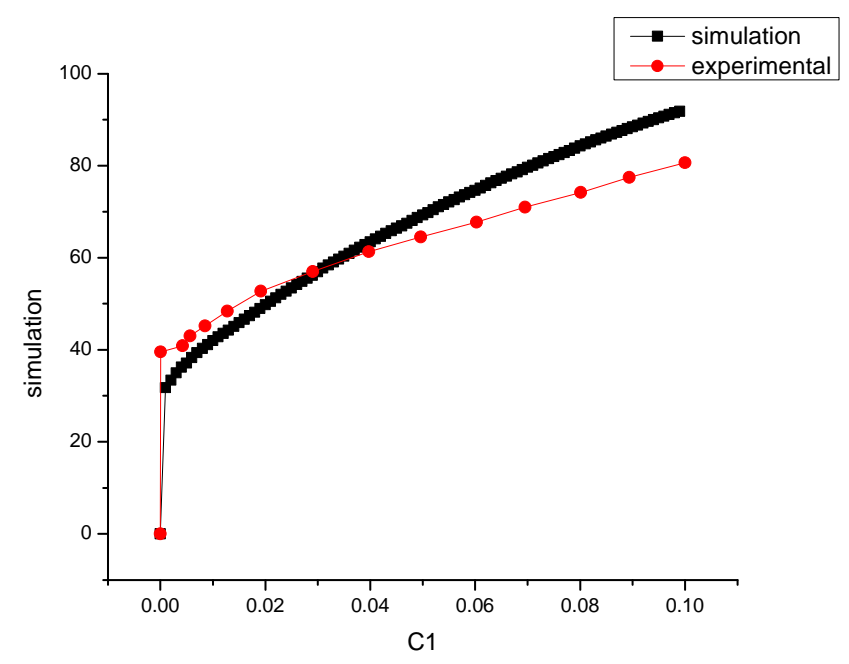

Figure 13. Measured and predicted stress strain curve of single crystal $\alpha$-Fe under uniaxial tension along $<011>$ direction.

Figure 13 shows the predicted and experimental results of single crystal $\alpha$-Fe with loading direction along the crystal orientation $<011>$. The continuum DD also demonstrated success in this crystal orientation. 
The strength and deformation behavior presented herein are only for single crystal cases. We are implementing our crystal plasticity in polycrystalline materials to incorporate the predicted results from continuum DD. In the developing framework, the influence of texture and grain boundary will be taken into consideration. Continuum DD also will be applied to irradiation hardening of steel. Likewise, the interaction between the dislocation and defects will be considered.

\section{SUMMARY AND CONCLUSIONS}

We conducted simulations of the motion of an edge dislocation at various stress levels and temperatures to produce functional mobility laws that can be used in large-scale DD analyses. Three systems were studied: the pure $\mathrm{Fe}$ and two $\mathrm{Fe}$ alloys, $\mathrm{Fe}-\mathrm{Ni}$ and $\mathrm{Fe}-\mathrm{Cr}$, with various concentrations of $\mathrm{Ni}$ and $\mathrm{Cr}$ to identify the effect of the solute concentration on dislocation mobility. Our results reveal the dependence of the dislocation mobility stemming from the temperature and concentration of the solute. A power form was used to fit the computational results. The fitting parameters were found either to vary linearly or remain constant. This observation allows us to form universal laws for the mobility as a function of concentration. These laws are in a form used in the DD framework to study the mechanical behavior of the systems at higher length and time scales. Furthermore, the results discussed in this work for edge dislocations can be coupled with findings for screw dislocations presented elsewhere by other authors, forming a unified framework that will describe the mobility of a dislocation inside pure Fe and Fe systems. The continuum DD proposed in this work is based on dislocation evolution mechanisms used in discrete DD. Using the information from the discrete DD, the continuum DD demonstrated success in predicting strength and deformation behavior of single crystal $\alpha-F e$. With the consideration of anisotropic Peierls stress and cross slip, the continuum DD is a model with high fidelity in predicting strength and deformation behavior of polycrystalline structural materials in nuclear engineering. 


\section{ACKNOWLEDGEMENTS}

This work was funded by DOE's Nuclear Energy Advanced Modeling and Simulation (NEAMS) program at Pacific Northwest National Laboratory (PNNL). PNNL is operated by Battelle for the DOE under contract No. DE-AC05-76RL01830. 


\section{REFERENCES}

[1] A Technology Roadmap for Generation IV Nuclear Energy Systems. U.S. DOE Nuclear Energy Research Advisory Committee and the Generation IV International Forum, 2002.

[2] Guerin Y, Was GS, Zinkle SJ. Materials Challanges for Advanced Nuclear Energy Systems. MRS Bulletin 2009;34:10.

[3] Klueh RL. Elevated-Temperature Ferritic and Martensitic Steels and their Application to Future Nuclear Reactors. Oak Ridge National Laboratory Report No. ORNL/TM-2004/176

2004.

[4] Klueh RL, Harris DR. High-Chromium Ferritic and Martensitic Steels for Nuclear Applications. 2001;ASTM International, West Conshohocken, PA

[5] Gelles DS. Microstructural development in reduced activation ferritic alloys irradiated to 200 dpa at $420^{\circ} \mathrm{C}$ J. Nuclear Engineering 1994;212-215:714.

[6] Gelles DS. Microstructural examination of commercial ferritic alloys at 200 dpa J.Nuc. Mater. 1996;233-237:293.

[7] Vitek JM, Corwin WR, Klueh RL, Howthorne JR. On the saturation of the DBTT shift of irradiated 12Cr-1MoVW with increasing fluence. J. Nucl. Mater. 1986;141-143:948.

[8] Khabarov VS, Dvoriashin, Porollo SI. Microstructure, irradiation hardening and embrittlement of $13 \mathrm{Cr} 2 \mathrm{MoNbVB}$ ferritic - martensitic steel after neutron irradiation at low temperatures J. Nucl. Mater. 1996;233-237:236.

[9] Zinkle SJ, Ghoniem NM. Operating temperature windows for fusion reactor structural materials Fusion Engineering \& Design;51-52:55.

[10] Odette GR. Modeling of microstructural evolution under irradiation. J. Nucl. Mater. 1979;8586:544.

[11] Gelles DS. Swelling in several commercial alloys irradiated to very high neutron fluence. J. Nucl. Mater. 1984;122:207.

[12] Wolfer WG. Advances in void swelling and helium bubble physics. J. Nucl. Mater. 1984;122:367.

[13] Singh BN, Foreman AJE. Relative role of gas generation and displacement rates in cavity nucleation and growth J.Nuc. Mater. 1984;122:537.

[14] Trinkaus H, Singh BN. Helium accumulation in metals during irradiation - where do we stand. J.Nuc. Mater. 2003;323:229.

[15] Singh, B.N., Ghoniem NM, Trinkaus H. Experiment-based modelling of hardening and localized plasticity in metals irradiated under cascade damage conditions J. Nucl. Mater. 2002;307-311:159.

[16] Mansur LK, Maziasz PJ, Rowcliffe AP. Control of helium effects in irradiated materials based on theory and experiment. J.Nuc. Mater. 1986;141-143:633.

[17] Spatig P, Schaublin R, Gyger S, Victoria M. Evolution of the mechanical properties of the F82H ferritic/martensitic steel after $590 \mathrm{MeV}$ proton irradiation 1989;258-263:1354.

[18] Gelles DS, Rice PM, Zinkle SJ, Chung HM. Microstructural examination of irradiated V-(45\%)Cr-(4-5\%)Ti J.Nuc. Mater. 1989;258-263:1380. 
[19] Li M, Hashimota N, Byun TS, Zinkle SJ. Defect cluster formation and radiation hardening in molybdenum neutron-irradiated at $80{ }^{\circ} \mathrm{C}$ J.Nuc. Mater. 2007;367-370:817.

[20] Golubov SI, Stoller RE, Zinkle SJ, Ovcharenko AM. Kinetics of coarsening of helium bubbles during implantation and post-implantation annealing J.Nuc. Mater. 2007;361:149.

[21] Wakai E, Ando M, Sawai T, Tanigawa H, Taguchi T, Stoller RE, Yamamoto T, Kato Y, Takada F. Effect of heat treatments on tensile properties of F82H steel irradiated by neutrons J.Nuc. Mater. 2007;367-370:74.

[22] Kobayashi S, Kikuchi H, Takahashi S, Kamada Y, Ara K, Yamamoto T, Klingensmith D, Odette GR. The effect of copper and manganese on magnetic minor hysteresis loops in neutron irradiated $\mathrm{Fe}$ model alloys J.Nuc. Mater. 2009;384:109.

[23] Zinkle SJ, Singh BN. Microstructure of neutron-irradiated iron before and after tensile deformation J.Nuc. Mater. 2006;351:269.

[24] Kurtz RJ, Alamo A, Lucon E, Huang Q, Jitsukawa S, Kimura A, Klueh RL, Odette GR, Petersen C, Sokilov MA, Rensman J-W. Recent progress toward development of reduced activation ferritic/martensitic steels for fusion structural applications J.Nuc. Mater. 2009;Available online 11 January 2009.

[25] Spatig P, Bonade R, Odette GR, Rensman J-W, Campitelli EN, Mueller P. Plastic flow properties and fracture toughness characterization of unirradiated and irradiated tempered martensitic steels J.Nuc. Mater. 2007;367-370:527.

[26] Hoelzer DT, Bentley J, Sokolov MA, Odette GR, Alinger MJ. Influence of particle dispersions on the high-temperature strength of ferritic alloys J.Nuc. Mater. 2007;367-370:166.

[27] Yamamoto T, Odette GR, Miao P, Hoelzer DT, Bentley J, Hashimota N, Tanigawa H, Kurtz RJ. The transport and fate of helium in nanostructured ferritic alloys at fusion relevant $\mathrm{He} / \mathrm{dpa}$ ratios and dpa rates J.Nuc. Mater. 2007;367-370:399.

[28] Yang L, Zu X, Wang Z, Gao G, Wang X, Heinisch H, Kurtz R. Interaction of helium-vacancy clusters with edge dislocations in alpha-Fe. Nucl Instrum Meth B 2007;265:541.

[29] Demkowicz MJ, Hoagland RG, Hirth JP. Interface structure and radiation damage resistance in $\mathrm{Cu}-\mathrm{Nb}$ multilayer nanocomposites. Phy. Review Letters 2008;100:136102.

[30] Diaz de la Rubia T, Zbib HM, Victoria M, Wright A, Khraishi T, Caturla M. Flow Localization in Irradiated Materials: A Multiscale Modeling Approach. Nature 2000;406:871.

[31] Zbib HM, Rhee M, Hirth JP. 3D Simulation of Curved Dislocations: Discretization and Long Range Interactions. Advances in Engineering Plasticity and its Applications, eds. T. Abe and T. Tsuta. Pergamon, NY 1996:15.

[32] Zbib HM, Rhee M, Hirth JP. On Plastic Deformation and the Dynamcis of 3D Dislocations. International Journal of Mechanical Science 1998;40:113.

[33] Canova G, Brechet Y, Kubin LP, Devincre B, Pontikis V, Condat M. 3D Simulation of Dislocation Motion on a Lattice: Application to the Yield Surface of Single Crystals. Microstructures and Physical Properties (ed. J. Rabiet), CH-Transtech 1993.

[34] Ghoniem NM, Sun L. A Fast Sum Method for the Elastic Field of 3-D Dislocation Ensembles. Phys. Rev. B, 1999;60:128.

[35] Khraishi T, Zbib HM, Diaz de la Rubia T, Victoria M. Modeling of Flow Localization and Hardening In Irradiated Materials Using Discrete Dislocation Dynamics (DD). Acta. Metall 2000. 
[36] Khraishi T, Zbib HM, Diaz de la Rubia T, Victoria M. Localized Deformation and Hardening In Irradiated Metals: Three-Dimensional Discrete Dislocation Dynamics Simulations,. Metall. Mater. Trans. 2002;33B:285.

[37] Zbib HM, de La Rubia TD, Rhee M, Hirth JP. 3D Dislocation Dynamics: Stress-Strain behavior and Hardening Mechanisms in FCC and BCC Metals. J. Nuc. Maters 2000;276:154.

[38] Hiratani M, Zbib HM, Wirth B. Interaction of Glissele Dislocations with Perfect and Truncated Stacking Fault Tetraherda in Irradiated Materials. Phil Mag. 2002;82:2709.

[39] Hiratani M, Zbib HM, Khaleel MA. Modeling of Thermally Activated Dislocation Glide and Plastic Flow through Local Obstacles. Int. J. Plasticity 2002;19:1271.

[40] Li DS, Zbib HM, Garmestani HS, Khaleel M, Sun X. Modeling of irradiation hardening of Polycrystalline Materials. Comp. Mater. \& Cont. 2010;50:2496.

[41] Ghoniem NM, Singh BN, Sun LZ, de la Rubia TD. Interaction and accumulation of glissile defect clusters near dislocations. Journal of Nuclear Materials 2000;276:166.

[42] Gordon PA, Neeraj T, Li L, Li J. crew dislocation mobility in BCC metals: the role of the compact core on double-kink nucleation. Modelling and Simulation in Materials Science and Engineering 2010;18:085008.

[43] Ngan AH, Wen M. Atomistic simulation of energetics of motion of screw dislocations in bcc Fe at finite temperatures. Comput. Mater. Sci 2002;23:139.

[44] Cai W, Chang J, Li J, Yip S, editors. Dislocation core effects on mobility. Amsterdam: Elsevier, 2004.

[45] Gilbert MR, Queyreau S, Marian J. Stress and temperature dependence of screw dislocation mobility in $\alpha$-Fe by molecular dynamics. Phys. Rev. B 2011;84:174103.

[46] Wang ZQ, Beyerlein IJ. An atmositically-informed dislocation dynamics model for the plastic anisotropy and tension-compression asymmetry of bec metals. Int. J. Plasticity 2011;27:1471.

[47] Mastorakos I, Zbib HM, Khaleel M. The effect of copper and nickel concentration on the mobility of edge dislocations in Fe alloys. Mat Sc Eng A 2011;submitted.

[48] Mastorakos I, Le L, Zeine M, Zbib HM, Khaleel M. Multiscale Modeling of irradiation induced hardening. In: $\alpha-\mathrm{Fe}, \mathrm{Fe}-\mathrm{Cr}$ and $\mathrm{Fe}-\mathrm{Ni}$ Systems. In: $\alpha-\mathrm{Fe}, \mathrm{Fe}-\mathrm{Cr}$ and $\mathrm{Fe}-\mathrm{Ni}$ Systems, in Basic Actinide Science and Materials for Nuclear Applications. In: J.K. Gibson SKM, E.D. Bauer, L. Soderholm, T. Fanghaenel, R. Devanathan, A. Misra, C. Trautmann, B.D. Wirth, editor. MRS, vol. 1264: MRS, 2010.

[49] Hirth JP, Lothe J. Theory of Dislocations. New York: Wiley, 1982.

[50] Mastorakos I, Zbib HM, Khaleel M. On the interaction of an edge dislocation with spherical voids in $\alpha$-Fe and Fe-Ni systems. Computational Materials Science 2011; submitted.

[51] Li DS, Zbib H, Garmestani H, Sun X, Khaleel M. A Hierarchical Upscaling Method for Predicting Strength of Materials under Thermal, Radiation and Mechanical loading - Irradiation Strengthening Mechanisms in Stainless Steels. Richland: Pacific Northwest National Laboratory, 2011.

[52] Daw M, Baskes M. Embedded-atom method: Derivation and application to impurities, surfaces, and other defects in metals. Physical Review 1983;B 29:6443.

[53] Mendelev MI, Han S, Srolovitz DJ, Ackland GJ, Sun DY, Asta M. Development of new interatomic potentials appropriate for crystalline and liquid iron. Phil Mag 2003;83:3977. 
[54] Stukowski S, Sadigh B, Erhart P, Caro A. Efficient implementation of the concentrationdependent embedded atom method for molecular-dynamics and Monte-Carlo simulations. Modelling and Simulation in Materials Science and Engineering, 2009;17:075005.

[55] Bonny G, Pasianot C, Malerba L. e-Ni many-body potential for metallurgical applications. Modelling and Simulation in Materials Science and Engineering 2009;17:075005.

[56] Zbib HM, de la Rubia TD. A multiscale model of plasticity. International Journal of Plasticity 2002;18:1133.

[57] Kawanishi H, Kuramoto E. J. Nuc. Mater. 1986;899:141.

[58] Khraishi TA, Zbib HM, De La Rubia TD, Victoria M. Localized deformation and hardening in irradiated metals: Three-dimensional discrete dislocation dynamics simulations. Metallurgical and Materials Transactions B-Process Metallurgy and Materials Processing Science 2002;33:285.

[59] Hiratani M, Bulatov V, Zbib HM. Orientation dependent elastic interaction between a truncated stacking fault tetrahedron and a glissile dislocation. . J. Nuc. Materials, 2004;329-333:1103.

[60] Guyot P, Dorn JE. A critical review on the Peierls mechanism. Can. J. Phys. 1967;45:983.

[61] Nadgorny E. Dislocation Dynamics and Mechanical Properties of Crystals. Oxford: Pergamon Press, 1988.

[62] Zinkle SJ, Matsukawa Y. Observation and analysis of defect cluster production and interactions with dislocations. Journal of Nuclear Materials 2004;329:88.

[63] Kocks U. Theory of an Obstacle-Controlled Yield Strength--Report After an International Workshop. Mater. Sci. Eng. 1977;27:291.

[64] Seeger A, Diehl J, Mader S, Rebstock H. Work-hardening and work-softening of face-centred cubic metal crystals Philosophical Magazine 1957;2:323.

[65] Seeger A, Diehl J, Mader S, Rebstock H. Work-hardening and work-softening of face-centred cubic metal crystals. Phil Mag 1957;2:323.

[66] Singh B, Evans J. Significant differences in defect accumulation behaviour between fcc and bcc crystals under cascade damage conditions. J. Nuc Mater. 1995;226:277.

[67] Trinkaus H, Singh BN, Foreman AJE. Mechanism for decoration of disloctions by small disloction loops under cascade damage conditions. J. Nuc Maters. 1997;249:91.

[68] Arsenlis A, Wirth BD, Rhee M. Disloction density-based constitutive model for the mechanical bahaviour of irradiated Cu. Phil Mag 2004;84:3517.

[69] Peeters B, Kalidindi S, Van Houtte P, Aernoudt E. A crystal plasticity based workhardening/softening model for bcc metals under changing strain paths. Acta materialia 2000;48:2123.

[70] Schoenfeld S, Ahzi S, Asaro R. Elastic-plastic crystal mechanics for low symmetry crystals. Journal of the Mechanics and Physics of Solids 1995;43:415.

[71] Orowan E. Problems of plastic gliding. Proceedings of the Physical Society 1940;52:8.

[72] Ohashi T. Numerical modeling of plastic multislip in metal crystals of fcc type. Philos. Mag. APhys. Condens. Matter Struct. Defect Mech. Prop. 1994;70:793.

[73] Ohashi T, Kawamukai M, Zbib H. A multiscale approach for modeling scale-dependent yield stress in polycrystalline metals. International Journal of Plasticity 2007;23:897.

[74] Kocks UF. Laws for work-hardening and low-temperature creep. Journal of Engineering Materials and Technology-Transactions of the Asme 1976;98:76. 
[75] Lee MG, Lim H, Adams BL, Hirth JP, Wagoner RH. A dislocation density-based single crystal constitutive equation. International Journal of Plasticity 2010;26:925.

[76] Lim H, Lee MG, Kim JH, Adams BL, Wagoner RH. Simulation of polycrystal deformation with grain and grain boundary effects. International Journal of Plasticity 2011;27:1328.

[77] Keh AS. Work hardening and deformation sub-structure in iron single crystals deformed in tension at 298K. Philosophical Magazine 1965;12:9. 